\title{
Integrated bioinformatics analysis of the NEDD4 family reveals a prognostic value of NEDD4L in clear-cell renal cell cancer
}

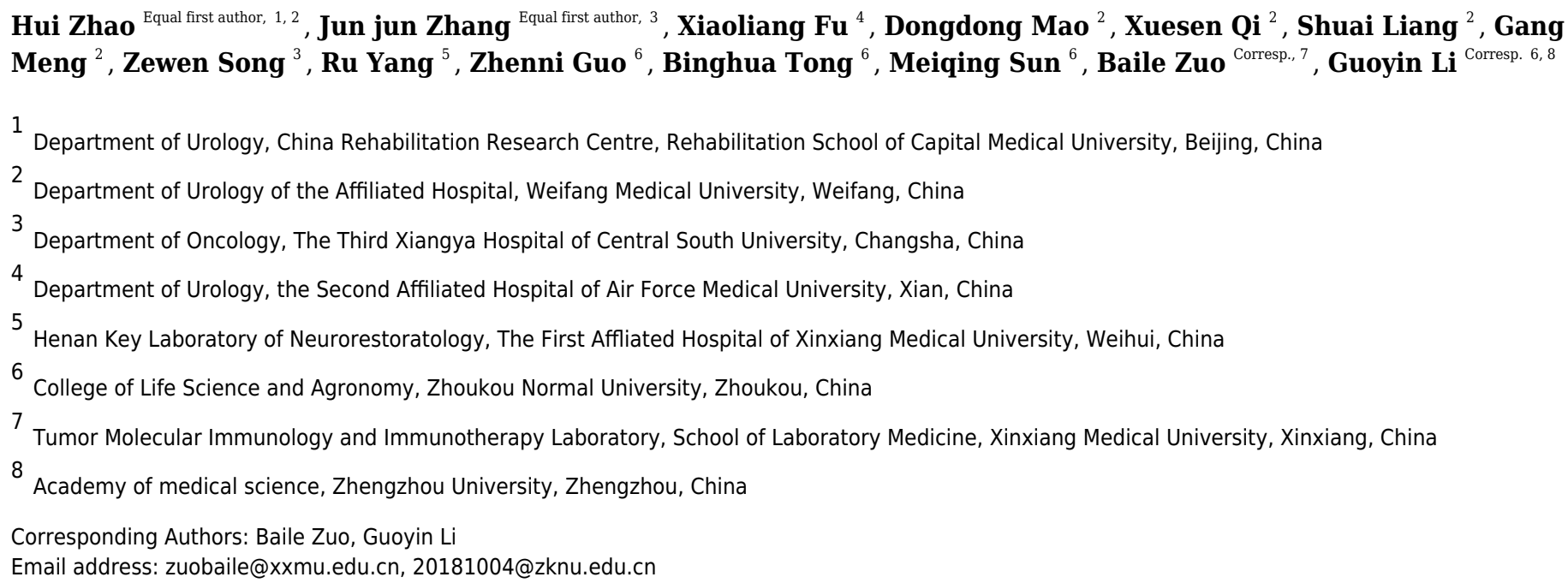

The members of the Nedd4-like E3 family participate in various biological processes. However, its role in clear cell renal cell carcinoma (ccRCC) is not clear. This study systematically analyzed the Nedd4-like E3 family members in cCRCC data sets from multiple publicly available databases. NEDD4L was identified as the only NEDD4 family member differentially expressed in ccRCC compared with normal samples. Bioinformatics tools were used to characterize the function of NEDD4L in CCRCC. It indicated that NEDD4L might regulate cellular energy metabolism by co-expression analysis, and subsequent gene ontology (GO) and Kyoto Encyclopedia of Genes and Genomes (KEGG) analysis . A prognostic model developed by the LASSO Cox regression method showed a relatively good predictive value in training and testing data sets. The result revealed that NEDD4L was associated with biosynthesis and metabolism of cCRCC. Since NEDD4L is downregulated and dysregulation of metabolism is involved in tumor progression, NEDD4L might be a potential therapeutic target in ccRCC. 


\section{Integrated Bioinformatics Analysis of the NEDD4 Family}

\section{Reveals a Prognostic Value of NEDD4L in Clear-cell Renal Cell}

3

\section{Cancer}

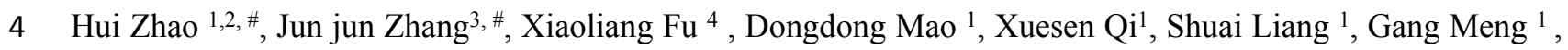

5 Zewen Song ${ }^{3}$, Ru Yang ${ }^{5}$, Zhenni Guo ${ }^{6}$, Binghua Tong ${ }^{6}$, Meiqing Sun ${ }^{6}$, Baile Zuo ${ }^{7, *}$, Guoyin $\mathrm{Li}^{8,6,}$,

6 'Department of Urology of the Affiliated Hospital, Weifang Medical University, Weifang, 261031, China

$7 \quad{ }^{2}$ Department of Urology, China Rehabilitation Research Centre, Rehabilitation School of Capital Medical University, Beijing,

8 100068, China

$9{ }^{3}$ Department of Oncology, The Third Xiangya Hospital of Central South University, Changsha 410013, China

$10{ }^{4}$ Department of Urology, the Second Affiliated Hospital of Air Force Medical University, Xian, 710038, China

$11{ }^{5}$ Henan Key Laboratory of Neurorestoratology, The First Affliated Hospital of Xinxiang Medical University, Weihui, 453100,

12 China.

${ }^{6}$ College of Life Science and Agronomy, Zhoukou Normal University, Zhoukou, 466000, China

${ }^{7}$ Tumor Molecular Immunology and Immunotherapy Laboratory, School of Laboratory Medicine, Xinxiang Medical University,

Xinxiang, 453003, China

${ }^{8}$ Academy of medical science, Zhengzhou University, Zhengzhou, 450001, China

${ }^{\#}$ These authors contributed equally to this work. 
27

28

29

\section{Abstract}

The members of the Nedd4-like E3 family participate in various biological processes. However, its role in clear cell renal cell carcinoma (ccRCC) is not clear. This study systematically analyzed the Nedd4-like E3 family members in ccRCC data sets from multiple publicly available databases. NEDD4L was identified as the only NEDD4 family member differentially expressed in ccRCC compared with normal samples. Bioinformatics tools were used to characterize the function of NEDD4L in ccRCC. It indicated that NEDD4L might regulate cellular energy metabolism by co-expression analysis, and subsequent gene ontology (GO) and Kyoto Encyclopedia of Genes and Genomes (KEGG) analysis. A prognostic model developed by the LASSO Cox regression method showed a relatively good predictive value in training and testing data sets. The result revealed that NEDD4L was associated with biosynthesis and metabolism of ccRCC. Since NEDD4L is downregulated and dysregulation of metabolism is involved in tumor progression, NEDD4L might be a potential therapeutic target in ccRCC.

Keywords: NEDD4L; bioinformatics; clear-cell renal cell cancer; prognosis; metabolism 


\section{Introduction}

Renal cell carcinoma (RCC) is the third most common malignant tumor in the urinary system(Bray et al., 2018). Recently the incidence of RCC has been increasing, and approximately 403,262 new cases and 175,098 kidney cancer-related deaths occurred worldwide in 2018(Siegel et al., 2019). Clear-cell renal cell carcinoma (ccRCC) is the most common pathological type and accounts for about two-thirds of all RCCs(Kroeger et al., 2013). Nearly 30$40 \%$ of patients with ccRCC will develop metastases(Ghatalia et al., 2019; Pierorazio et al., 2016). The Von Hippel-Lindau/hypoxia-inducible factor (VHL/HIF) axis, the major carcinogenic pathway in ccRCC, promotes angiogenesis, cell growth and glycolysis by activating vascular endothelial growth factor A (VEGFA), transforming growth factor alpha and beta and platelet derived growth factor(Seles et al., 2020; Sanchez-Gastaldo et al., 2017; Maxwell et al., 1999). Compared to chemotherapy and radiotherapy, surgical resection of early-stage renal cancer is more effective(Chow et al., 2010). However, 20-40\% of cases will relapse after surgery(Janzen et al., 2003). Accordingly, it is necessary to find biomarkers that can be effectively used to monitor RCC progression and evaluate its prognosis. Research shows that E3 ubiquitin ligases take part in various biological processes involved in the pathogenesis of carcinoma which could be considered as potential biomarker candidates(Wang et al., 2017).

The Nedd4-like E3 family mediates ubiquitination and proteasomal degradation of its substrate proteins as well as receptor-mediated endocytosis. In mammals, there are nine members: NEDD4, NEDD4L, ITCH, SMURF1, SMURF2, WWP1, WWP2, NEDL1 and NEDL2(Wang et al., 2020). The Nedd4-like E3 family members have similar domains, including an N terminal C2 calcium-dependent phospholipid binding domain, 3-4 WW protein-protein interaction domains, and a C-terminal catalytic HECT ubiquitin ligase domain(Boase \& Kumar, 2015). The Nedd4like E3 family plays an important role in various biological processes. In recent years, the role of the NEDD4 family in urinary system tumors has attracted much attention. In bladder cancer, NEDD4 exerts its oncogenic function by regulating phosphatase and tensin homolog (PTEN) and notch receptor 1 (NOTCH1) expression(Wen et al., 2017). In prostate cancer, NEED4 exerts its 
oncogenic function by downregulating PTEN and androgen receptor (AR)(Li et al., 2015; Li et al., 2008). NEDD4L is down-regulated in prostate cancer, and may exert an antitumor function by regulating TGF $\beta 1$ signaling(Li et al., 2015; Li et al., 2008). However, research on the function of NEDD4 family members in ccRCC is lacking, and more attention needs to be paid to this topic.

The expression level of $N E D D 4 L$ has been found significantly decreased in various tumors(Tanksley et al., 2013a; Yang et al., 2015a; Hu et al., 2009b; Gao et al., 2012; Zhao et al., 2015a). It is reported that NEDD4L acts a pivotal part in the prognosis of various malignant carcinomas(Jiang et al., 2019a). Also, NEDD4L has been shown to be involved in the regulation of certain major signaling pathways in carcinoma, including the WNT, and TGF- $\beta$ signaling pathways(Zou et al., 2015). In addition, NEDD4L has been shown to act as a cancer suppressor by inhibiting canonical WNT signaling in colorectal cancer(Tanksley et al., 2013a). Additionally, NEDD4L was found to suppress tumor metastasis by inhibiting the activity of TGF- $\beta$ signaling pathway(Kuratomi et al., 2005). NEDD4L can bind to TGFBRI via SMAD7 and induce its degradation through ubiquitin proteasome pathway, thereby preventing the activation of RSMADs. Moreover, NEDD4L can bind to SMAD2 and SMAD3 in a ligand-dependent manner and induce the degradation of SMAD2(Kuratomi et al., 2005). However, NEDD4L was confirmed to have distinct functions in different carcinomas. Furthermore, NEDD4L was found to exert its pro-oncogenic function in gallbladder cancer, where it promotes invasion by regulating transcription of the matrix metallopeptidase 1 and 13 genes(Takeuchi et al., 2011). However, the function of NEDD4L in ccRCC remains unknown.

Fatty acids metabolism exerts new functions in cancer, such as protecting cells from lipotoxicity, facilitating cell migration and drug resistance by altering membrane fluidity, and meeting high energy demands of metastatic cells through fatty acid $\beta$-oxidation (FAO)Tumor progression depends on the reprogramming of cell metabolism(Pavlova \& Thompson, 2016). In recent years, elevated lipid synthesis is considered to be an important metabolic abnormality for tumorigenesis(Swierczynski et al., 2014). The biosynthesis of fatty acids is increased, and the 
103

104

105

106

107

108

109

110

111

112

113

114

115

116

117

118

119

120

121

122

123

124

125

126

127

128

129

metabolism of fatty acids exerts new functions in cancer, such as protecting cells from lipotoxicity, facilitating cell migration and drug resistance by altering membrane fluidity, and meeting high energy demands of metastatic cells through fatty acid $\beta$-oxidation (FAO)(Chen \& Huang, 2019b). The rapid proliferation of tumor cells also increases their demand for amino acids. In addition to being used for protein synthesis, amino acids can also be used as metabolites and regulators to support the growth of tumor cells(Li \& Zhang, 2016).

There are not many researches on NEDD4L and cell metabolism, which need more attention from researchers. In this paper, we integrated data from multiple public databases like the Cancer Genome Atlas (TCGA), Oncomine and gene expression database (GEO) to methodically study the expression profile, prognostic significance and role of NEDD4L in ccRCC. The results indicate that NEDD4L is markedly downregulated in ccRCC, which is related to poor prognosis. Enrichment analysis indicates that NEDD4L may regulate biosynthesis of unsaturated fatty acids and fatty acids metabolism. These findings reveal for the first time the effect of NEDD4L in the prognosis of ccRCC and the potential involvement of this protein in the regulation of fatty acid synthesis and metabolism.

\section{Materials and methods}

\section{Data acquisition and processing}

The kidney clear cell Carcinoma (KIRC) project of the TCGA (TCGA_KIRC) data set and GEO data sets (GSE40435 and GSE53757) were acquired and processed using the method described in our previous study (Zhang et al., 2020). Briefly, normalized RNA-seq data (607 cases, HTSeq-FPKM), phenotype information (985 cases), and survival data (979 cases) of the TCGA_KIRC were downloaded from the GDC hub of UCSC xena website (http://xena.ucsc.edu/public). Gene expression data of the GSE40435 and GSE53757 data sets (series matrix file) was downloaded from the Gene Expression Omnibus (GEO) database through the GEOquery package in the $\mathrm{R}$ software (version 3.6.2). After data processing, 526 tumor samples with survival data of the TCGA_KIRC data set, 202 samples of the GSE40435, and 144 samples of the GSE53757 were used for further analysis. All data for this study were acquired 
130

131

132

133

134

135

136

137

138

139

140

141

142

143

144

145

146

147

148

149

150

151

152

153

154

155

156

from public databases and did not require approval from the Ethics Committee.

\section{Online database analysis}

The transcriptional level of NEDD4L in renal cell carcinoma was verified in the Oncomine database (https://www.oncomine.org/resource/login.html). The expression data of 5 groups of renal cell carcinoma and normal tissues (transformed by $\log 2$ ) were retrieved and compared statistically. The default threshold was as follows: $\mathrm{P}<1 \mathrm{E}^{-4}$, multiple change $>2$, and the top $10 \%$ of genes. The staining intensity of NEDD4L in RCC samples and normal kidneys were downloaded from the Human Protein Atlas database (https://www.proteinatlas.org/).

\section{Functional analysis and enrichment analysis}

Gene ontology (GO) and Kyoto Encyclopedia of Genes and Genomes (KEGG) enrichment analyses were carried out using the clusterProfiler package in R version 3.6.2(Hsing et al., 2020).

Gene set enrichment analysis (GSEA) was used to investigate pathways enriched in the high- and low-risk subgroups. C2.cp.kegg.v7.1.symbols.gmt was chosen as the gene set database. These pathways were considered to be significantly enriched when the following criteria were met: nominal $\mathrm{p}$-value $<0.05$, false discovery rate $\mathrm{q}$-value $<0.25$, and absolute normalized enrichment score $>1$.

\section{Development of the prognostic model}

The least absolute shrinkage and selection operator (LASSO) Cox regression analysis was performed using the glmnet package in $\mathrm{R}$. The analysis generated key gene signatures, and their corresponding coefficients were obtained by multi-variate cox analysis. A new score was calculated by multiplying the gene expression value of each gene and their corresponding coeffecient, as follows:

Score $=-0.06919 *$ SOWAHB $-0.18041 *$ WDR72 - 0.22124*EPB41L4A-DT - 0.07547*C4orf19 $0.22609 *$ CDKL2 - 0.05303*IRF6 - 0.16642*DHRS7 - 0.31446*NUPR2.

The risk score was calculated by subtracting the minimum score of the cohort from this score, and dividing the difference by the absolute value of the maximum score of the cohort, namely riskscore $=($ Score-min $($ Score $)) / \operatorname{abs}(\max ($ Score $))$. 
157

158

159

160

161

162

163

164

165

166

167

168

169

170

171

172

173

174

175

176

177

178

179

180

181

182

183

\section{Development and evaluation of the nomogram}

The independent risk factors determined by multivariate Cox regression analysis were chosen to develop a nomogram for prediction of total overall survival (OS) probability. The consistency index (C-index) was used to evaluate the consistency between the occurrence frequency of the actual results and the prediction probability of the model. The nomogram was generated using the rms package in R software(Li et al., 2019).

\section{Statistical analysis}

The data collected were analyzed by default as described using web resources. The rest of the data was analyzed using $\mathrm{R}$ software (version 3.6.2). The patients were divided into two subgroups by the median value of gene expression or risk score. Briefly, the correlation between the expression of NEDD4L and the clinicopathological features of ccRCC patients was examined by chi-square test with SPSS software (IBM Corp., Armonk, NY, USA). The expression of NEDD4L and other genes was analyzed by Spearman correlation analysis with $\mathrm{R}$ software (version 3.6.2). Students't-test was used to compare the level of gene expression. Univariate/multivariate Cox regression were performed by using the 'survminer' package in R. Kaplan-Meier survival analysis was used to compare the OS, relapse-free survival (RFS), disease specific survival (DSS) and progression free interval (PFI) of high- and low-CIFI groups Time-dependent receiver operator characteristic (ROC) analyses and subsequent calculation of the area under the curve (AUC)(Le NQK, 2019; Le NQK et al., 2019) were performed using the 'timeROC' package in R. Packages in R used for data analysis and graph plotting included ggstatsplot, ggplot2, ggpubr, limma, survminer, survival, tidyverse, dplyr, and plyr. A value of $p$ $<0.05$ was considered to be statistically significant $(*, p<0.05 ; * *, p<0.01 ; * * *, p<0.001$; $* * * *, p<0.0001)$.

\section{Results}

\section{Patient characteristics}

The data were collected from the TCGA_KIRC data set, including 72 normal subjects and 530 patients with primary tumors with clinical and gene expression data. The 526 patients with 
184

185

186

187

188

189

190

191

192

193

194

195

196

197

198

199

200

201

202

203

204

205

206

207

208

209

survival information were used for follow-up analysis (Supplementary Table 1). There were 183 females $(34.79 \%)$ and 343 males $(65.21 \%)$. The onset age ranged from 26 to 88 years old, and the median age was 60 years old, $245(46.58 \%)$ cases were younger than 60 years, and 281 $(53.42 \%)$ cases were older than 60 years. The classification of the tumors according to the TNM (tumor, node, metastasis) staging system was as follows: 267 (50.76\%) cases with T1 stage and $259(49.24 \%)$ cases with T2-4 stage; 238 (45.25\%) cases with N0 stage and $288(54.75 \%)$ cases with N1/X stage; 418 (79.47\%) cases with M0 stage, 106 (20.15\%) cases with N1/X stage, and 2 $(0.38 \%)$ cases of unknown stage; 239 (45.44\%) cases with G1/2 stage, 279 (53.04\%) cases with G3/4, and $8(1.52 \%)$ cases of unknown stage. The classification of the tumors according to the Overall Stage Grouping (OSG) was as follows: 318 (60.46\%) cases with stage I / II, 205 (38.97\%) cases with stage III/IV, and $3(0.57 \%)$ cases of unknown stage; $330(60.46 \%)$ cases with stage I / II , 147 (38.97\%) cases with stage III/IV, and 49 (0.57\%) cases of unknown stage. The GSE40435 (101 normal subjects and 101 primary tumors), and GSE53757 (70 normal subjects and 70 primary tumors) data sets from the GEO database were used for verification.

\section{NEDD4L is downregulated in ccRCC}

We screened the transcription levels of NEDD4 family members in the TCGA_KIRC. The differentially expressed genes (DEGs) between normal and tumor tissues were analyzed using the following criteria: fold change $(\mathrm{FC})>2$ and $\mathrm{FDR}<0.05$. The results revealed that only $N E D D 4 L$ was meaningfully down-regulated in tumors compared with that in normal tissues (Figure 1A). Subsequently, two data sets from the GEO database were used for verification, and the results confirmed that $N E D D 4 L$ was significantly down-regulated in ccRCC carcinoma tissues (Figure 1B\&1C). Data from the Oncomine database made up of five separate queues also confirmed that NEDD4L was meaningfully down-regulated in ccRCC (Figure 1D). Immunohistochemical results showed that the expression level of NEDD4L in renal cell carcinoma was significantly decreased than that in normal kindey (Supplementary Figure 1).

\section{Low expression of NEDD4L correlated to dismal prognosis in ccRCC}


The TCGA_KIRC data set was used to investigate the prognostic value of NEDD4L in

211

212

213

214

215

216

217

218

219

220

221

222

223

224

225

226

227

228

229

230

231

232

233

234

235

236

ccRCC. After data filtering, a total of 526 samples with gene expression and survival data were included. The median of the gene expression was set as the cut-off value. Kaplan-Meier survival analysis showed that patients with low NEDD4L expression had shorter OS $(\mathrm{P}<0.0001)$, PFS (P $<0.0001)$ and DSS $(P<0.0001)$ (Figure 1E-1G). Patients were divided into different subgroups according to sex, age, laterality, cancer status, histological grade, TNM stage, and OSG tumor stage. Analysis results of all but the N1 and M1 subgroups showed that patients with high NEDD4L expression had longer OS (Figure 2\&3, Supplementary Figure 2\&3). Univariate and multivariate analysis were used to validate the prognostic value of NEDD4L in ccRCC. The univariate analysis results demonstated that low NEDD $4 L$ expression was associated with shorter OS (hazard ratio [HR]: 2.323; 95\% confidence interval [CI]: 1.683-3.208; $P=0.001$ ) (Table 1). Multivariate COX regression analysis revealed that low expression of NEDD4L was still an independent factor associated with the deterioration of OS (HR: 1.905; CI: 1.33-2.73, P $<0.001$ ) (Table 1). These results suggested that NEDD4L may be an independent prognostic factor for ccRCC.

\section{NEDD4L downregulation correlated with worse clinicopathological features of ccRCC}

To investigate the relationship between the expression of NEDD4L and clinicopathological features in renal cell carcinoma, patients were divided into low and high expression subgroup on the base of the median expression of NEDD4L. Chi-square test showed that lower levels of NEDD4L were significantly associated with adverse prognostic features, including ccRCC with higher T stages $(\mathrm{OR}=0.456(0.322-0.646)$ for $\mathrm{T} 1$ vs. T2/3/4, $P<0.001), \mathrm{M}$ stage $(\mathrm{OR}=0.530$ (0.342-0.821) for M0 vs. M1/X, $P=0.004)$, OSG tumor stage $(\mathrm{OR}=0.429(0.299-0.615)$ for stage I / II vs. stage III/IV, $P<0.001)$, histological grade $(\mathrm{OR}=0.456(0.321-0.647)$ for G1/2 vs. G3/4, $P<0.001)$ (table 2).

\section{Co-expression and enrichment analysis}

Since NEDD4L was downregulated in ccRCC and correlated with worse prognosis of patients with ccRCC, we undertook to elucidate its potential role in this disease. In TCGA_KIRC 
237

238

239

240

241

242

243

244

245

246

247

248

249

250

251

252

253

254

255

256

257

258

259

260

261

262

263

and GSE40435 data sets, the correlation between the expression of NEDD4L and other genes in tumor samples was analyzed. A total of 494 genes in the TCGA_KIRC and 515 genes in the GSE40435 were identified as NEDD4L-related genes, using the following criteria: coefficient > 0.5 and $\mathrm{p}$ value $<0.05$. Among them, 206 NEDD4L-related genes were shared between the two datasets (Supplementary Figure 4, Supplementary Table 2) and were subjected to GO and KEGG analysis. GO analysis revealed that the proteins encoded by these genes were mainly located in mitochondrial membrane and matrix, involved in the catabolism of many metabolites, such as lipid oxidation, fatty acid oxidation, fatty acid catabolism, monocarboxylic acid catabolism, and performed various molecular functions, such as DNA binding and ATP enzyme activity (Fig. 4A, Supplementary Table 3). Accordingly, KEGG analysis showed that these genes play a major role in amino acid metabolism, oxidative phosphorylation and citrate cycle (figure 4B). These results suggest that NEDD4L is closely related to ccRCC cell metabolism.

\section{Development of a NEDD4L-related prognostic model}

To develop a NEDD4L-related prognostic model for ccRCC, we first performed univariate Cox regression analysis on the aforementioned 206 NEDD4L-related genes, which identified 183 genes with significant prognostic relevance $(\mathrm{p}<0.05$, Supplementary Table 2$)$. Patients from TCGA_KIRC $(\mathrm{n}=526)$ were randomly and equally divided into two groups, one is to establish a LASSO Cox regression model, and the other is to evaluate the effectiveness of the model. The 183 NEDD4L-related genes with significant prognostic relevance were input into a LASSO Cox regression model, which generated eight key genes (Figure 5A\&5B), namely DHRS7, NUPR2, C4orf19, CDKL2, SOWAHB, WDR72, EPB41L4A-DT, and IRF6 (Figure 5C). The risk score was calculated by inputting the selected signature genes into the above formula. The median risk score was set to a cutoff value, dividing patients into low-risk subgroups and high-risk subgroups. Prognostic analysis with Kaplan-Meier method showed that patients with ccRCC in the low risk subgroup had significantly longer DSS (Figure 6A-6C). The analysis of the time-related receiver operating characteristic (ROC) in the TCGA training data set shows that the risk score had a favorable predictive value (Figure 6D, area under the curve (AUC) at 1 year $=0.72$, AUC at 3 
264 years $=0.75$, AUC at 5 years $=0.8$ ). The predictions of the established risk scores were further 265 confirmed by the TCGA test data set and the entire TCGA data set. The ROC analysis of the 266 TCGA testing dataset showed that the AUC was 0.76 at 1 year, 0.69 at 3 years, and 0.68 at 5 267 years (Figure 6E), while that of the TCGA entire dataset showed that the AUC was 0.74 at 1 year,

268

269

270

271

272

0.71 at 3 years, and 0.74 at 5 years (Figure 6F). As reflected by the AUC values at 1 year, 3 year and 5 years, the signatures developed in our work had relatively better performance in predicting outcome of ccRCC patients (Figure 6F, Supplementary Figure 5)(Zhang et al., 2021; Fei et al., 2021; Chu et al., 2021; Xing et al., 2021b).

\section{Identification of NEDD4L-related signaling pathways by GSEA}

Various biological processes are recognized to be involved in the occurrence and development of ccRCC. Accordingly, we hypothesized that the worse survival related to low expression of NEDD4L may be associated with some activated signal pathways in ccRCC. To evaluate this hypothesis, we performed a GSEA of the differences in the NEDD4L-related low risk group in TCGA_KIRC to verify the key signaling pathways related to NEDD4L expression. There were significant differences in the richness of MSigDB sets (c2.cp.v6.2 symbols) of multiple pathways (normalized $\mathrm{P}<0.05, \mathrm{FDR}<0.25$ ). According to the normalized enrichment score (NES), the signaling pathways most significantly associated with NEDD4L expression are shown in Figure 7 and Table 3. In particular, NEDD4L expression was associated with biosynthesis of unsaturated fatty acids, insulin signaling pathway, and fatty acid metabolism, supporting a possible role of NEDD4L in the regulation of various metabolic processes

\section{Establishment of a nomogram based on NEDD4L}

Univariate and multivariate Cox regression analysis were achieved on the TCGA_KIRC ccRCC data set to investigate whether the risk score was an independent predictor of prognosis in patients with ccRCC. The adjustment of conventional clinical models, including age, sex, laterality, TNM stage, cancer status, histological grade, and tumor stage, indicated that the risk score was also an independent prognostic factor, which confirmed it had a strong ability to predict $\mathrm{OS}$ in patients with $\mathrm{ccRCC}(\mathrm{OR}=1.9329(1.3429-2.782), P<0.001$, Figure 8A\&8B) 
291

292

293

294

295

296

297

298

299

300

301

302

303

304

305

306

307

308

309

310

311

312

313

314

315

316

317

To establish a quantitative method for ccRCC prognosis, we used independent clinical risk factors to develop a nomogram (Figure 8C). The point scale of nomogram is used to assign points to each variable based on multivariate Cox analysis. We drew a horizontal line to determine the points of each variable, then added the points of all variables together to calculate the total score of each patient, and then normalize it to a distribution of 0 to 45 . The estimated 1 -, 3-and 5-year survival rates of ccRCC patients were calculated by drawing a vertical line between the total point axis and each prognostic axis(Li et al., 2019). The C-index of the nomogram $0.7627102(0.7255857-0.7998348)$ suggesting a good prediction effect. Thus, our results prove that nomogram was the best model for predicting the prognosis of renal cell carcinoma compared with a single risk factor.

\section{Discussion}

The Nedd4-like E3 family includes nine members, which regulate key signaling pathways in tumorigenesis, such as TGF $\beta$, EGF, IGF, VEGF, CXCL12, and TNF(Chen \& Matesic, 2007). In cancers, NEDD4, WWP1, WWP2, SMURF1, and SMURF2 have been shown to function as oncogenes, while NEDD4L acts as an antioncogene(Chen \& Matesic, 2007; Tanksley et al., 2013b; Zhao et al., 2015b). This study systematically investigated the role of the NEDD4 family in ccRCC through the comprehensive analysis of TCGA_KIRC and GEO data sets and Oncomine database. The analysis revealed that only NEDD4L was significantly downregulated in ccRCC, and low expression of NEDD4L was associated with shorter survival (Figure 1).

NEDD4 is shown to be overexpressed in various types of human cancers including gastric cancer(Kim et al., 2008; Sun et al., 2014; Reichert-Penetrat et al., 1998), colorectal cancer(Kim et al., 2008), breast cancer(Kim et al., 2008), non-small-cell lung carcinoma(Amodio et al., 2010; Shao et al., 2018), and hepatocellular carcinoma(Hang et al., 2016; Huang et al., 2017). NEDD4 acts as a double-edged sword in tumors. On the one hand, NEDD4 promotes tumor survival by inducing cell proliferation, inhibiting apoptosis, disrupting the cell cycle, promoting cell migration and invasion, and enhancing drug resistance(Wang et al., 2020). On the other hand, NEDD4 inhibits tumor proliferation by binding to MYC, inhibiting HER3 expression, and 
318

319

320

321

322

323

324

325

326

327

328

329

330

331

332

333

334

335

336

337

338

339

340

341

342

343

344

targeting PIP5K1A. SMURFs also plays a dual role in tumors, both as tumor promoters and suppressors(David et al., 2013). By controlling the stability of several key proteins, these proteins play a central role in cell cycle progression, proliferation, differentiation, metastasis, genome stability and senescence. WWP1 is frequently upregulated in multiple human malignancies, and has been found to play an important role in cell proliferation, apoptosis and invasion(Nourashrafeddin et al., 2015; Zhi \& Chen, 2012; Chen et al., 2007; Chen et al., 2007; Chen \& Zhang, 2018). WWP2 functions as an oncogene in liver cancer, breast cancer, endometrial cancer, gastric cancer, lung cancer, oral cancer, and ovarian cancer. The expression of NEDL1 in benign neuroblastoma is higher than that in non-malignant neuroblastoma(Li et al., 2008). So far as we know, there are no reportes on ITCH and NEDL2 in tumors, thus their function in ccRCC is unclear. Our study showed that their expression levels were no significantly changed in ccRCC, which is inconsistent with reports in other tumors, which may be due to tumor specificity.

NEDD4L has been considered as a tumor suppressor. Decreased NEDD4L expression has been found in colorectal cancer(Tanksley et al., 2013c), gastric cancer(Jiang et al., 2019b), ovarian cancer(Yang et al., 2015b), prostate cancer(Hu et al., 2009c), and non-small cell lung cancer(Hu et al., 2009c). Consistent with previous reports, this study suggested that NEDD4L acts as a tumor suppressor in ccRCC. Subsequent analysis showed that NEDD4L is an independent prognostic factor for ccRCC and might be associated with energy metabolism. Lee et al. reported that NEDD4L inhibited autophagy and mitochondrial metabolism by downregulating the expression of ULK1 or ASCT2, thereby inhibiting the growth and survival of pancreatic cancer cells. Kim et al. reported that NEDD4L acts a pivotal part in the feedback regulation of cAMP signal by limiting CREB-regulated transcription coactivator 3 protein levels(Kim et al., 2018). The findings of the above studies are consistent with the findings of our study, indicating that NEDD4L plays an important role in tumor metabolism.

We also established a prognostic model based on NEDD4L using the LASSO Cox regression, a broadly selected machine learning algorithm used to minimize the risk of 
345

346

347

348

349

350

351

352

353

354

355

356

357

358

359

360

361

362

363

364

365

366

367

368

369

370

371

overfitting. The model had good predictive value for training and test queues (Figure 6). A total of 526 cases from TCGA_KIRC were randomly divided into low-risk and high-risk subgroups, and then analyzed by GSEA, GO, and KEGG enrichment analysis, which are widely used bioinformatics tools in the functional characterization of specific genes.

All of these enrichment analyses suggested that the main difference between the two groups is in metabolism (Figure 4\&7, Table 3). The decreased expression of NEDD4L may lead to metabolic disorder and promote the development of ccRCC.

Lee et al.'s study found that NEDD4L could inhibit the growth and survival of pancreatic cancer cells by inhibiting autophagy and mitochondrial metabolism(Lee et al., 2020). Zhang et al. reported that NEDD4L also played vital roles in bone metabolism. These studies provided strong evidence for the involvement of NEDD4L in cell metabolism (PMID: 22957059). The prediction model developed in this study showed that DHRS7, NUPR2, C4orf19, CDKL2, SOWAHB, WDR72, EPB41L4A_DT, and IRF6 were significantly associated with NEDD4L function.

DHRS7 can recognize steroids and retinoids as potential substrates, and might act a pivotal part in the metabolism of these signal molecules(Stambergova et al., 2016). Lopez et al. reported that NUPR2 could induce G1 phase cell cycle arrest, decrease cell survival rate and proliferation ability(Lopez et al., 2015). C4orf19 was found to be significantly downregulated in multicentric breast cancer, but its function is unclear(Lang et al., 2017). Two studies showed that the decreased expression of CDKL2 was associated with the progression and poor prognosis of gliomas(Yi et al., 2018; Yi et al., 2020), and another study showed that CDKL2 promoted EMT and progression of breast cancer(Li et al., 2014). WDR72 was found to be crucial in ameloblasts and the kidneys, and may act a pivotal part in enamel maturation(Rungroj et al., 2018). EPB41L4A_DT was reported to inhibit the progression of ovarian cancer, hepatocellular carcinoma, non-small cell lung cancer, and breast cancer(Sun et al., 2020; Wang et al., 2019; Shu et al., 2018; Xu et al., 2016). IRF6 has been proved to be a tumor suppressor in breast and gastric cancer(Li et al., 2019; Xu et al., 2019). All these genes play important roles in a variety of solid tumors, indirectly confirming the importance of NEDD4L in ccRCC. 
A NEDD4L-related prognostic model of ccRCC was established by LASSO Cox regression.

373

374

375

8

After adjusting the routine clinical features, the risk score was confirmed as an independent prognostic factor. It seemed that the risk score may have a stronger predictive power than the traditional prognostic factors. We also conducted a comprehensive assessment in combination with risk scores and other important clinical models. According to the calibration graph, there was a significant consistency between the actual and predicted values of the 1 -, 3-and 5-year OS (figure 8). In order to show the reliability of our risk model, we compared it with other ccRCC risk models, and the results showed that our risk model has better prediction ability (Supplementary Figure 5)(Zhang et al., 2021; Fei et al., 2021; Chu et al., 2021; Xing et al., 2021). Based on the complementary perspective of the corresponding tumor, the model provides a personalized score for individual patients and a valuable new prognostic evaluation method for clinicians.

Although this study provides new clues for the relationship between NEDD4L expression and ccRCC progression, it still has some limitations. First, it is necessary to further verify the function of NEDD4L in ccRCC and cellular metabolic processes, and completely elucidate the specific mechanism involved. Second, our risk score requires a large sample of prospective studies to be demonstrated, which is just a retrospective analysis.

\section{Acknowledgments}

This work was supported by the National Natural Science Foundation of China (81903031), China Postdoctoral Science Foundation (2020M682334), Henan Postdoctoral Foundation (202003002), the Open Project of State Key Laboratory of Cancer Biology of China (CBSKL2019KF12, CBSKL2019KF13). We thank Editorbar (www.editorbar.com) for providing language assistance in the preparation of this manuscript.

\section{Disclosure}

The authors declare no conflicts of interest in this work.

(1)

\section{References:}

Amodio, N., Scrima, M., Palaia, L., Salman, A.N., Quintiero, A., Franco, R., Botti, G., Pirozzi, P., Rocco, G., De 
400 Rosa, N., and Viglietto, G. 2010. Oncogenic role of the E3 ubiquitin ligase NEDD4-1, a PTEN negative regulator, in

401 non-small-cell lung carcinomas. AMERICAN JOURNAL OF PATHOLOGY 177:2622-2634.

402 10.2353/ajpath.2010.091075

403 Boase, N.A., and Kumar, S. 2015. NEDD4: The founding member of a family of ubiquitin-protein ligases. GENE 404 557:113-122. 10.1016/j.gene.2014.12.020

405 Bray, F., Ferlay, J., Soerjomataram, I., Siegel, R.L., Torre, L.A., and Jemal, A. 2018. Global cancer statistics 2018: 406 GLOBOCAN estimates of incidence and mortality worldwide for 36 cancers in 185 countries. CA Cancer J Clin 407 68:394-424. 10.3322/caac.21492

408 Butt, G., Yaylim, I., Attar, R., Aras, A., Romero, M.A., Qureshi, M.Z., Purenovic, J., and Farooqi, A.A. 2019. 409 NEDD4 Family of E3 Ubiquitin Ligases in Breast Cancer: Spotlight on SMURFs, WWPs and NEDD4. Advances 410 in Experimental Medicine and Biology 1152:365-375. 10.1007/978-3-030-20301-6_19

411 Chen, C., Sun, X., Guo, P., Dong, X.Y., Sethi, P., Zhou, W., Zhou, Z., Petros, J., Frierson, H.J., Vessella, R.L., Atfi, 412 A., and Dong, J.T. 2007. Ubiquitin E3 ligase WWP1 as an oncogenic factor in human prostate cancer. ONCOGENE 413 26:2386-2394. 10.1038/sj.onc.1210021

414 Chen, C., Zhou, Z., Ross, J.S., Zhou, W., and Dong, J.T. 2007. The amplified WWP1 gene is a potential molecular 415 target in breast cancer. INTERNATIONAL JOURNAL OF CANCER 121:80-87. 10.1002/ijc.22653

416 Chen, C., and Matesic, L.E. 2007. The Nedd4-like family of E3 ubiquitin ligases and cancer. Cancer Metastasis Rev 417 26:587-604. 10.1007/s10555-007-9091-x

418 Chen, J.J., and Zhang, W. 2018. High expression of WWP1 predicts poor prognosis and associates with tumor 419 progression in human colorectal cancer. American Journal of Cancer Research 8:256-265.

420 Chen, M., and Huang, J. 2019b. The expanded role of fatty acid metabolism in cancer: new aspects and targets. 421 Precis Clin Med 2:183-191. 10.1093/pcmedi/pbz017

422 Chow, T.F., Youssef, Y.M., Lianidou, E., Romaschin, A.D., Honey, R.J., Stewart, R., Pace, K.T., and Yousef, G.M. 423 2010. Differential expression profiling of microRNAs and their potential involvement in renal cell carcinoma 424 pathogenesis. CLINICAL BIOCHEMISTRY 43:150-158. 10.1016/j.clinbiochem.2009.07.020

425 Chu, G., Xu, T., Zhu, G., Liu, S., Niu, H., and Zhang, M. 2021. Identification of a Novel Protein-Based Signature to 426 Improve Prognosis Prediction in Renal Clear Cell Carcinoma. Front Mol Biosci 8:623120. 427 10.3389/fmolb.2021.623120

428 Clements, A.E., Bravo, V., Koivisto, C., Cohn, D.E., and Leone, G. 2015. WWP2 and its association with PTEN in 429 endometrial cancer. Gynecol Oncol Rep 13:26-29. 10.1016/j.gore.2015.05.004

430 David, D., Nair, S.A., and Pillai, M.R. 2013. Smurf E3 ubiquitin ligases at the cross roads of oncogenesis and tumor 431 suppression. Biochim Biophys Acta 1835:119-128. 10.1016/j.bbcan.2012.11.003

432 Fang, S., Zhang, D., Weng, W., Lv, X., Zheng, L., Chen, M., Fan, X., Mao, J., Mao, C., Ye, Y., Xu, M., and Ji, J. 433 2020. CPSF7 regulates liver cancer growth and metastasis by facilitating WWP2-FL and targeting the 434 WWP2/PTEN/AKT signaling pathway. Biochim Biophys Acta Mol Cell Res 1867:118624. 435 10.1016/j.bbamcr.2019.118624

436 Fei, H., Chen, S., and Xu, C. 2021. Construction autophagy-related prognostic risk signature combined with 437 clinicopathological validation analysis for survival prediction of kidney renal papillary cell carcinoma patients. $B M C$ 438 CANCER 21:411. 10.1186/s12885-021-08139-2

439 Fukumoto, C., Nakashima, D., Kasamatsu, A., Unozawa, M., Shida-Sakazume, T., Higo, M., Ogawara, K., Yokoe, 440 H., Shiiba, M., Tanzawa, H., and Uzawa, K. 2014. WWP2 is overexpressed in human oral cancer, determining 
441

442

443

444

445

446

447

448

449

450

451

452

453

454

455

456

457

458

459

460

461

462

463

464

465

466

467

468

469

470

471

472

473

474

475

476

477

478

479

480

481

tumor size and poor prognosis in patients: downregulation of WWP2 inhibits the AKT signaling and tumor growth in mice. Oncoscience 1:807-820. 10.18632/oncoscience.101

Gao, C., Pang, L., Ren, C., and Ma, T. 2012. Decreased expression of Nedd4L correlates with poor prognosis in gastric cancer patient. MEDICAL ONCOLOGY 29:1733-1738. 10.1007/s12032-011-0061-3

Ghatalia, P., Gordetsky, J., Kuo, F., Dulaimi, E., Cai, K.Q., Devarajan, K., Bae, S., Naik, G., Chan, T.A., Uzzo, R., Hakimi, A.A., Sonpavde, G., and Plimack, E. 2019. Prognostic impact of immune gene expression signature and tumor infiltrating immune cells in localized clear cell renal cell carcinoma. Journal for ImmunoTherapy of Cancer 7:139. 10.1186/s40425-019-0621-1

Hang, X., Zhu, S., Di H, Wu, Z., Chu, K., Wang, J., Xin, H., Yu, G., Peng, H., Miao, X., and Xu, W. 2016. NEDD4 Depletion Inhibits Hepatocellular Carcinoma Growth via Targeting PTEN. CELLULAR PHYSIOLOGY AND BIOCHEMISTRY 39:768-779. 10.1159/000445667

Hsing, M., Wang, Y., Rennie, P.S., Cox, M.E., and Cherkasov, A. 2020. ETS transcription factors as emerging drug targets in cancer. MEDICINAL RESEARCH REVIEWS 40:413-430. 10.1002/med.21575

Hu, X.Y., Xu, Y.M., Fu, Q., Yu, J.J., and Huang, J. 2009a. Nedd4L expression is downregulated in prostate cancer compared to benign prostatic hyperplasia. European Journal of Surgical Oncology (EJSO) 35:527-531. 10.1016/j.ejso.2008.09.015

Hu, X.Y., Xu, Y.M., Fu, Q., Yu, J.J., and Huang, J. 2009b. Nedd4L expression is downregulated in prostate cancer compared to benign prostatic hyperplasia. Eur J Surg Oncol 35:527-531. 10.1016/j.ejso.2008.09.015

Hu, X.Y., Xu, Y.M., Fu, Q., Yu, J.J., and Huang, J. 2009c. Nedd4L expression is downregulated in prostate cancer compared to benign prostatic hyperplasia. Eur J Surg Oncol 35:527-531. 10.1016/j.ejso.2008.09.015

Huang, Z.J., Zhu, J.J., Yang, X.Y., and Biskup, E. 2017. NEDD4 promotes cell growth and migration via PTEN/PI3K/AKT signaling in hepatocellular carcinoma. Oncology Letters 14:2649-2656. 10.3892/ol.2017.6532

Janzen, N.K., Kim, H.L., Figlin, R.A., and Belldegrun, A.S. 2003. Surveillance after radical or partial nephrectomy for localized renal cell carcinoma and management of recurrent disease. Urol Clin North Am 30:843-852. 10.1016/s0094-0143(03)00056-9

Jiang, X., Zhang, S., Yin, Z., Sheng, Y., Yan, Q., Sun, R., Lu, M., Zhang, Z., and Li, Y. 2019a. The correlation between NEDD4L and HIF-1alpha levels as a gastric cancer prognostic marker. International Journal of Medical Sciences 16:1517-1524. 10.7150/ijms.34646

Jiang, X., Zhang, S., Yin, Z., Sheng, Y., Yan, Q., Sun, R., Lu, M., Zhang, Z., and Li, Y. 2019b. The correlation between NEDD4L and HIF-1alpha levels as a gastric cancer prognostic marker. International Journal of Medical Sciences 16:1517-1524. 10.7150/ijms.34646

Jiang, X., Zhang, S., Yin, Z., Sheng, Y., Yan, Q., Sun, R., Lu, M., Zhang, Z., and Li, Y. 2019c. The correlation between NEDD4L and HIF-1alpha levels as a gastric cancer prognostic marker. International Journal of Medical Sciences 16:1517-1524. 10.7150/ijms.34646

Jung, J.G., Stoeck, A., Guan, B., Wu, R.C., Zhu, H., Blackshaw, S., Shih, I., and Wang, T.L. 2014. Notch3 interactome analysis identified WWP2 as a negative regulator of Notch3 signaling in ovarian cancer. PLoS Genetics 10:e1004751. 10.1371/journal.pgen.1004751

Kim, S.S., Yoo, N.J., Jeong, E.G., Kim, M.S., and Lee, S.H. 2008. Expression of NEDD4-1, a PTEN regulator, in gastric and colorectal carcinomas. APMIS 116:779-784. 10.1111/j.1600-0463.2008.00999.x

Kim, Y.H., Yoo, H., Hong, A.R., Kwon, M., Kang, S.W., Kim, K., and Song, Y. 2018. NEDD4L limits cAMP signaling through ubiquitination of CREB-regulated transcription coactivator 3. FASEB JOURNAL 32:4053-4062.

Peer) reviewing PDF | (2021:02:57683:2:0:NEW 18 Jun 2021) 
482

483

484

485

486

487

488

489

490

491

492

493

494

495

496

497

498

499

500

501

502

503

504

505

506

507

508

509

510

511

512

513

514

515

516

517

518

519

520

521

522

\subsection{6/fj.201701406R}

Kroeger, N., Xie, W., Lee, J.L., Bjarnason, G.A., Knox, J.J., Mackenzie, M.J., Wood, L., Srinivas, S., Vaishamayan, U.N., Rha, S.Y., Pal, S.K., Yuasa, T., Donskov, F., Agarwal, N., Kollmannsberger, C.K., Tan, M.H., North, S.A., Rini, B.I., Choueiri, T.K., and Heng, D.Y. 2013. Metastatic non-clear cell renal cell carcinoma treated with targeted therapy agents: characterization of survival outcome and application of the International mRCC Database Consortium criteria. CANCER 119:2999-3006. 10.1002/cncr.28151

Kuratomi, G., Komuro, A., Goto, K., Shinozaki, M., Miyazawa, K., Miyazono, K., and Imamura, T. 2005. NEDD42 (neural precursor cell expressed, developmentally down-regulated 4-2) negatively regulates TGF-beta (transforming growth factor-beta) signalling by inducing ubiquitin-mediated degradation of Smad2 and TGF-beta type I receptor. BIOCHEMICAL JOURNAL 386:461-470. 10.1042/BJ20040738

Lang, Z.Q., Wu, Y.Q., Pan, X.B., Qu, G.M., and Zhang, T.G. 2017. The identification of multifocal breast cancerassociated long non-coding RNAs. Eur Rev Med Pharmacol Sci 21:5648-5654. 10.26355/eurrev_201712_14008

Le NQK. 2019. Fertility-GRU: Identifying Fertility-Related Proteins by Incorporating Deep-Gated Recurrent Units and Original Position-Specific Scoring Matrix Profiles. JOURNAL OF PROTEOME RESEARCH 18:3503-3511. 10.1021/acs.jproteome.9b00411

Le NQK, Yapp, E., Nagasundaram, N., and Yeh, H.Y. 2019. Classifying Promoters by Interpreting the Hidden Information of DNA Sequences via Deep Learning and Combination of Continuous FastText N-Grams. Front Bioeng Biotechnol 7:305. 10.3389/fbioe.2019.00305

Lee, D.E., Yoo, J.E., Kim, J., Kim, S., Kim, S., Lee, H., and Cheong, H. 2020. NEDD4L downregulates autophagy and cell growth by modulating ULK1 and a glutamine transporter. Cell Death \& Disease 11:38. 10.1038/s41419020-2242-5

Li, D., Cheng, P., Wang, J., Qiu, X., Zhang, X., Xu, L., Liu, Y., and Qin, S. 2019. IRF6 Is Directly Regulated by ZEB1 and ELF3, and Predicts a Favorable Prognosis in Gastric Cancer. Frontiers in Oncology 9:220. 10.3389/fonc.2019.00220

Li, G., Xu, W., Zhang, L., Liu, T., Jin, G., Song, J., Wu, J., Wang, Y., Chen, W., Zhang, C., Chen, X., Ding, Z., Zhu, P., and Zhang, B. 2019. Development and validation of a CIMP-associated prognostic model for hepatocellular carcinoma. EBioMedicine 47:128-141. 10.1016/j.ebiom.2019.08.064

Li, H., Mohamed, A.A., Sharad, S., Umeda, E., Song, Y., Young, D., Petrovics, G., McLeod, D.G., Sesterhenn, I.A., Sreenath, T., Dobi, A., and Srivastava, S. 2015. Silencing of PMEPA1 accelerates the growth of prostate cancer cells through AR, NEDD4 and PTEN. Oncotarget 6:15137-15149. 10.18632/oncotarget.3526

Li, H., Xu, L.L., Masuda, K., Raymundo, E., McLeod, D.G., Dobi, A., and Srivastava, S. 2008. A Feedback Loop between the Androgen Receptor and a NEDD4-binding Protein, PMEPA1, in Prostate Cancer Cells. JOURNAL OF BIOLOGICAL CHEMISTRY 283:28988-28995. 10.1074/jbc.M710528200

Li, L., Liu, C., Amato, R.J., Chang, J.T., Du G, and Li, W. 2014. CDKL2 promotes epithelial-mesenchymal transition and breast cancer progression. Oncotarget 5:10840-10853. 10.18632/oncotarget.2535

Li, Y., Ozaki, T., Kikuchi, H., Yamamoto, H., Ohira, M., and Nakagawara, A. 2008. A novel HECT-type E3 ubiquitin protein ligase NEDL1 enhances the p53-mediated apoptotic cell death in its catalytic activity-independent manner. ONCOGENE 27:3700-3709. 10.1038/sj.onc.1211032

$\mathrm{Li}$, Z., and Zhang, H. 2016. Reprogramming of glucose, fatty acid and amino acid metabolism for cancer progression. CELLULAR AND MOLECULAR LIFE SCIENCES 73:377-392. 10.1007/s00018-015-2070-4

Lopez, M.B., Garcia, M.N., Grasso, D., Bintz, J., Molejon, M.I., Velez, G., Lomberk, G., Neira, J.L., Urrutia, R.,

Peer) reviewing PDF | (2021:02:57683:2:0:NEW 18 Jun 2021) 
and Iovanna, J. 2015. Functional Characterization of Nupr1L, A Novel p53-Regulated Isoform of the High-Mobility Group (HMG)-Related Protumoral Protein Nupr1. JOURNAL OF CELLULAR PHYSIOLOGY 230:2936-2950. $10.1002 / \mathrm{jcp} .25022$

Maxwell, P.H., Wiesener, M.S., Chang, G.W., Clifford, S.C., Vaux, E.C., Cockman, M.E., Wykoff, C.C., Pugh, C.W., Maher, E.R., and Ratcliffe, P.J. 1999. The tumour suppressor protein VHL targets hypoxia-inducible factors for oxygen-dependent proteolysis. NATURE 399:271-275. 10.1038/20459

Nourashrafeddin, S., Aarabi, M., Modarressi, M.H., Rahmati, M., and Nouri, M. 2015. The Evaluation of WBP2NLRelated Genes Expression in Breast Cancer. PATHOLOGY \& ONCOLOGY RESEARCH 21:293-300. 10.1007/s12253-014-9820-8

Pavlova, N.N., and Thompson, C.B. 2016. The Emerging Hallmarks of Cancer Metabolism. Cell Metabolism 23:2747. 10.1016/j.cmet.2015.12.006

Pierorazio, P.M., Johnson, M.H., Patel, H.D., Sozio, S.M., Sharma, R., Iyoha, E., Bass, E.B., and Allaf, M.E. 2016. Management of Renal Masses and Localized Renal Cancer: Systematic Review and Meta-Analysis. J Urol 196:989999. 10.1016/j.juro.2016.04.081

Reichert-Penetrat, S., Barbaud, A., Martin, S., Omhover, L., Weber, M., and Schmutz, J.L. 1998. Pemphigus vulgaris on an old surgical scar: Koebner's phenomenon? EUROPEAN JOURNAL OF DERMATOLOGY 8:60-62.

Rungroj, N., Nettuwakul, C., Sawasdee, N., Sangnual, S., Deejai, N., Misgar, R.A., Pasena, A., Khositseth, S., Kirdpon, S., Sritippayawan, S., Vasuvattakul, S., and Yenchitsomanus, P.T. 2018. Distal renal tubular acidosis caused by tryptophan-aspartate repeat domain 72 (WDR72) mutations. CLINICAL GENETICS 94:409-418. $10.1111 /$ cge. 13418

Sakashita, H., Inoue, H., Akamine, S., Ishida, T., Inase, N., Shirao, K., Mori, M., and Mimori, K. 2013. Identification of the NEDD4L gene as a prognostic marker by integrated microarray analysis of copy number and gene expression profiling in non-small cell lung cancer. ANNALS OF SURGICAL ONCOLOGY 20 Suppl 3:S590S598. 10.1245/s10434-013-3059-6

Sanchez-Gastaldo, A., Kempf, E., Gonzalez, D.A.A., and Duran, I. 2017. Systemic treatment of renal cell cancer: A comprehensive review. CANCER TREATMENT REVIEWS 60:77-89. 10.1016/j.ctrv.2017.08.010

Seles, M., Hutterer, G.C., Fosselteder, J., Svoboda, M., Resel, M., Barth, D.A., Pichler, R., Bauernhofer, T., Zigeuner, R.E., Pummer, K., Slaby, O., Klec, C., and Pichler, M. 2020. Long Non-Coding RNA PANTR1 is Associated with Poor Prognosis and Influences Angiogenesis and Apoptosis in Clear-Cell Renal Cell Cancer. Cancers (Basel) 12. 10.3390/cancers12051200

Shao, G., Wang, R., Sun, A., Wei, J., Peng, K., Dai, Q., Yang, W., and Lin, Q. 2018. The E3 ubiquitin ligase NEDD4 mediates cell migration signaling of EGFR in lung cancer cells. Molecular Cancer 17:24. 10.1186/s12943018-0784-2

Shu, J., Li, S., Chen, Y.B., Zhu, Q.F., and Yu, X.H. 2018. Long non-coding RNA EPB41L4A-AS2 inhibited nonsmall cell lung cancer proliferation, invasion and promoted cell apoptosis. NEOPLASMA 65:664-672. 10.4149/neo_2018_170713N480

Siegel, R.L., Miller, K.D., and Jemal, A. 2019. Cancer statistics, 2019. CA Cancer J Clin 69:7-34. 10.3322/caac. 21551

Stambergova, H., Zemanova, L., Lundova, T., Malcekova, B., Skarka, A., Safr, M., and Wsol, V. 2016. Human DHRS7, promising enzyme in metabolism of steroids and retinoids? J Steroid Biochem Mol Biol 155:112-119. 10.1016/j.jsbmb.2015.09.041

Peer) reviewing PDF | (2021:02:57683:2:0:NEW 18 Jun 2021) 
564 Sun, A., Yu, G., Dou, X., Yan, X., Yang, W., and Lin, Q. 2014. Nedd4-1 is an exceptional prognostic biomarker for 565 gastric cardia adenocarcinoma and functionally associated with metastasis. Molecular Cancer 13:248. $56610.1186 / 1476-4598-13-248$

567 Sun, T., Yang, P., and Gao, Y. 2020. Long non-coding RNA EPB41L4A-AS2 suppresses progression of ovarian 568 cancer by sequestering microRNA-103a to upregulate transcription factor RUNX1T1. EXPERIMENTAL 569 PHYSIOLOGY 105:75-87. 10.1113/EP087847

570 Swierczynski, J., Hebanowska, A., and Sledzinski, T. 2014. Role of abnormal lipid metabolism in development, 571 progression, diagnosis and therapy of pancreatic cancer. World J Gastroenterol 20:2279-2303. 572 10.3748/wjg.v20.i9.2279

573 Takeuchi, T., Adachi, Y., Nagayama, T., and Furihata, M. 2011. Nedd4L modulates the transcription of 574 metalloproteinase-1 and -13 genes to increase the invasive activity of gallbladder cancer. INTERNATIONAL 575 JOURNAL OF EXPERIMENTAL PATHOLOGY 92:79-86. 10.1111/j.1365-2613.2010.00740.x

576 Tanksley, J.P., Chen, X., and Coffey, R.J. 2013a. NEDD4L is downregulated in colorectal cancer and inhibits 577 canonical WNT signaling. PLoS One 8:e81514. 10.1371/journal.pone.0081514

578 Tanksley, J.P., Chen, X., and Coffey, R.J. 2013b. NEDD4L is downregulated in colorectal cancer and inhibits 579 canonical WNT signaling. PLoS One 8:e81514. 10.1371/journal.pone.0081514

580 Tanksley, J.P., Chen, X., and Coffey, R.J. 2013c. NEDD4L is downregulated in colorectal cancer and inhibits 581 canonical WNT signaling. PLoS One 8:e81514. 10.1371/journal.pone.0081514

582 Wan, L., Liu, T., Hong, Z., Pan, Y., Sizemore, S.T., Zhang, J., and Ma, Z. 2019. NEDD4 expression is associated 583 with breast cancer progression and is predictive of a poor prognosis. BREAST CANCER RESEARCH 21:148. 584 10.1186/s13058-019-1236-7

585 Wang, D., Ma, L., Wang, B., Liu, J., and Wei, W. 2017. E3 ubiquitin ligases in cancer and implications for therapies. 586 Cancer Metastasis Rev 36:683-702. 10.1007/s10555-017-9703-z

587 Wang, K., Liu, J., Zhao, X., Li, H., Luo, G., Yu, Y., Guo, Y., Zhang, L., Zhu, J., Wang, S., Hua, W., Yang, A., 588 Zhang, R., and Li, J. 2020. WWP2 regulates proliferation of gastric cancer cells in a PTEN-dependent manner. 589 Biochem Biophys Res Commun 521:652-659. 10.1016/j.bbrc.2019.10.179

590 Wang, Y.G., Wang, T., Shi, M., and Zhai, B. 2019. Long noncoding RNA EPB41L4A-AS2 inhibits hepatocellular 591 carcinoma development by sponging miR-301a-5p and targeting FOXL1. J Exp Clin Cancer Res 38:153.

592 10.1186/s13046-019-1128-9

593 Wang, Z., Hu, X., Ye, M., Lin, M., Chu, M., and Shen, X. 2020. NEDD4 E3 ligase: Functions and mechanism in 594 human cancer. SEMINARS IN CANCER BIOLOGY 67:92-101. 10.1016/j.semcancer.2020.03.006

595 Wang, Z.W., Hu, X., Ye, M., Lin, M., Chu, M., and Shen, X. 2020. NEDD4 E3 ligase: Functions and mechanism in 596 human cancer. SEMINARS IN CANCER BIOLOGY 67:92-101. 10.1016/j.semcancer.2020.03.006

597 Wen, W., Li, J., Wang, L., Xing, Y., Li, X., Ruan, H., Xi, X., Xiong, J., and Kuang, R. 2017. Inhibition of NEDD4 598 inhibits cell growth and invasion and induces cell apoptosis in bladder cancer cells. CELL CYCLE 16:1509-1514. $59910.1080 / 15384101.2017 .1338220$

600 Xing, Q., Zeng, T., Liu, S., Cheng, H., Ma, L., and Wang, Y. 2021a. A novel 10 glycolysis-related genes signature 601 could predict overall survival for clear cell renal cell carcinoma. BMC CANCER 21:381. 10.1186/s12885-021$602 \quad 08111-0$

603 Xing, Q., Zeng, T., Liu, S., Cheng, H., Ma, L., and Wang, Y. 2021b. A novel 10 glycolysis-related genes signature 604 could predict overall survival for clear cell renal cell carcinoma. BMC CANCER 21:381. 10.1186/s12885-021- 


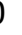

08111-0

Xu, H.F., Huang, T.J., Yang, Q., Xu, L., Lin, F., Lang, Y.H., Hu, H., Peng, L.X., Meng, D.F., Xie, Y.J., Tan, L., Qian, C.N., and Huang, B.J. 2019. Candidate tumor suppressor gene IRF6 is involved in human breast cancer pathogenesis via modulating PI3K-regulatory subunit PIK3R2 expression. Cancer Management and Research 11:5557-5572. 10.2147/CMAR.S203060

Xu, S., Wang, P., You, Z., Meng, H., Mu, G., Bai, X., Zhang, G., Zhang, J., and Pang, D. 2016. The long noncoding RNA EPB41L4A-AS2 inhibits tumor proliferation and is associated with favorable prognoses in breast cancer and other solid tumors. Oncotarget 7:20704-20717. 10.18632/oncotarget.8007

Yang, Q., Zhao, J., Cui, M., Gi, S., Wang, W., and Han, X. 2015a. Nedd4L expression is decreased in ovarian epithelial cancer tissues compared to ovarian non-cancer tissue. J Obstet Gynaecol Res 41:1959-1964. 10.1111/jog.12808

Yang, Q., Zhao, J., Cui, M., Gi, S., Wang, W., and Han, X. 2015b. Nedd4L expression is decreased in ovarian epithelial cancer tissues compared to ovarian non-cancer tissue. J Obstet Gynaecol Res 41:1959-1964. 10.1111/jog. 12808

Yi, R., Yang, S., Liao, Y., Hu, Z., Long, H., Zeng, Y., Wang, X., Qiu, C., Xu, A., Lin, J., and Wu, Z. 2020. Decreased CDKL2 expression is correlated with the progression and poor prognosis of glioma. PATHOLOGY RESEARCH AND PRACTICE 216:152920. 10.1016/j.prp.2020.152920

Yi, R., Yang, S., Wen, E., Hu, Z., Long, H., Zeng, Y., Wang, X., Huang, X., Liao, Y., Luo, M., Wang, J., Zhou, M., Wang, W., Xu, A., Lin, J., Wu, Z., and Song, Y. 2018. Negative nuclear expression of CDKL2 correlates with disease progression and poor prognosis of glioma. Int J Clin Exp Pathol 11:712-719.

Zhang, H., Qiu, X., and Yang, G. 2021. The CSRNP Gene Family Serves as a Prognostic Biomarker in Clear Cell Renal Cell Carcinoma. Frontiers in Oncology 11:620126. 10.3389/fonc.2021.620126

Zhang, J., Zhang, X., Li, J., and Song, Z. 2020. Systematic analysis of the ABC transporter family in hepatocellular carcinoma reveals the importance of ABCB6 in regulating ferroptosis. LIFE SCIENCES 257:118131. 10.1016/j.lfs.2020.118131

Zhao, R., Cui, T., Han, C., Zhang, X., He, J., Srivastava, A.K., Yu, J., Wani, A.A., and Wang, Q.E. 2015a. DDB2 modulates TGF-beta signal transduction in human ovarian cancer cells by downregulating NEDD4L. NUCLEIC ACIDS RESEARCH 43:7838-7849. 10.1093/nar/gkv667

Zhao, R., Cui, T., Han, C., Zhang, X., He, J., Srivastava, A.K., Yu, J., Wani, A.A., and Wang, Q.E. 2015b. DDB2 modulates TGF-beta signal transduction in human ovarian cancer cells by downregulating NEDD4L. NUCLEIC ACIDS RESEARCH 43:7838-7849. 10.1093/nar/gkv667

Zhi, X., and Chen, C. 2012. WWP1: a versatile ubiquitin E3 ligase in signaling and diseases. CELLULAR AND MOLECULAR LIFE SCIENCES 69:1425-1434. 10.1007/s00018-011-0871-7

Zou, X., Levy-Cohen, G., and Blank, M. 2015. Molecular functions of NEDD4 E3 ubiquitin ligases in cancer. Biochim Biophys Acta 1856:91-106. 10.1016/j.bbcan.2015.06.005

\section{Figure 1. Expression and prognostic analysis of NEDD4L in ccRCC}

(A) In TCGA_KIRC data set, DEGs were represented in a volcano plot. (B\&C) Mining of the GEO database showed that NEDD4L was downregulated in tumor tissues. (D) Data from the 
644 Oncomine database confirmed that NEDD4L was significantly downregulated in tumor tissues.

645 (E) Kaplan-Meier curves of the OS of the patients with ccRCC. (F) Kaplan-Meier curves of the 646 PFI of the patients with ccRCC. (G) Kaplan-Meier curves of the DSS of the patients with $647 \mathrm{ccRCC}$

648 Figure 2. Prognostic analysis of NEDD4L in ccRCC patients grouped by sex, age, laterality, 649 and cancer status

650 (A-D) Kaplan-Meier plot of the OS in high- and low-NEDD4L expression subgroups of ccRCC 651 patients who were grouped according to sex (A), age (B), laterality (C), cancer status (D)

652 Figure 3. Prognostic analysis of NEDD4L in ccRCC patients grouped by histological grade, 653 TNM stage, and tumor stage

654 (A-D) Kaplan-Meier plot of the OS in high- and low-NEDD4L expression subgroups of ccRCC 655 patients who were grouped according to histological grade (A), TNM stage (B\&C), and tumor 656 stage (D)

657 Figure 4. GO and KEGG enrichment analysis of NEDD4L

658 (A) GO enrichment analysis of NEDD4L. (B) KEGG enrichment analysis of 659 NEDD4L

660 Figure 5. Construction of LASSO Cox regression model

661 (A-C) A LASSO Cox regression model was developed from NEDD4L and 183 related genes, 662 calculating the tuning parameter $(\lambda)$ based on the partial likelihood deviance with tenfold cross663 validation. Each dot represents a patient, and the heatmap represents the genes expression levels. 664 The optimal $\log \lambda$ value is indicated by the vertical black line in the plot.

665 Figure 6. Reliability verification of NEDD4L-related prognostic model 666 (A-C) Kaplan-Meier plot of the DDS in the high- and low-risk groups of the TCGA_KIRC 667 cohort . (D-F) Time-dependent ROC analysis of the risk score for the DDS and survival status in 668 the TCGA_KIRC cohort

669 Figure 7. GSEA between ccRCC patients with high and low NEDD4L expression in the 670 TCGA_KIRC and GSE40435 cohorts (A-H). 
671 Figure 8. Integration of risk score and clinical characteristics

672 (A\&B) Univariate and multivariate regression analysis of the relation between the risk score and

673 clinicopathological characteristics regarding OS in the TCGA_KIRC. (C) Nomogram developed

674 to predict the 1-, 3-, and 5-year OS in the TCGA_KIRC data set

675 Supplementary Figure 1. Immunohistochemical results of NEDD4L.

676 Supplementary Figure 2. Kaplan-Meier plot of the OS in the high- and low-NEDD4L

677 expression subgroups of $\mathrm{M} 1 \mathrm{ccRCC}$ patients

678 Supplementary Figure 3. Kaplan-Meier plot of the OS in high- and low-NEDD4L

679 subgroups of N1 ccRCC patients

680 Supplementary Figure 4. Co-expression analysis of NEDD4L in TCGA_KIRC and

681 GSE40435 data sets.

682 Supplementary Figure 5. ROC curves for forecasting 1-year, 3-year and 5-year OS based

683 on risk score.

684 Table 1: Univariate and multivariate analysis of overall survival in TCGA patients with

685 ccRCC

686 Table 2 NEDD4L expression associated with clinical characteristics

687 Table 3 GSEA of the NEDD4L-related low risk group in TCGA_KIRC

688 Supplementary table 1: Clinical information of patients

689 Supplementary table 2: NEDD4L-related genes

690 Supplementary table 3: GO enrich of NEDD4L

691 


\section{Figure 1}

Figure 1. Expression and prognostic analysis of NEDD4L in CCRCC

(A) In TCGA_KIRC data set, DEGs were represented in a volcano plot. (B\&C) Mining of the GEO database showed that NEDD4L was downregulated in tumor tissues. (D) Data from the Oncomine database confirmed that NEDD4L was significantly downregulated in tumor tissues. (E) Kaplan-Meier curves of the OS of the patients with cCRCC. (F) Kaplan-Meier curves of the PFI of the patients with ccRCC. (G) Kaplan-Meier curves of the DSS of the patients with ccRCC 
A

DEGs of NEDD4 family in TCGA_KIRC change $\cdot$ DOWN $\cdot$ NOT $\cdot$ UP

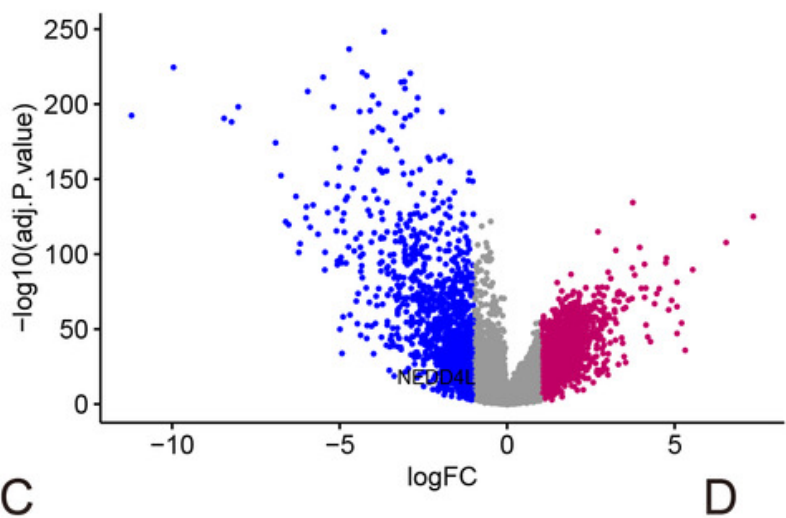

B

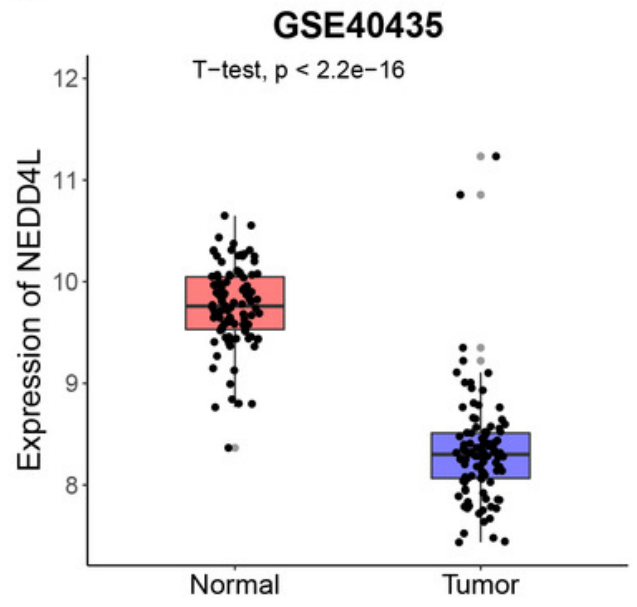

Comparison of NEDD4L Across 5 Analyses Under-expression

Median Rank p-Value Gene $226.0 \quad 3.23 E-5 \quad$ NEDD4L

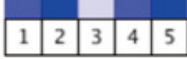

Legend

1. Clear Cell Renal Cell Carcinoma vs. Normal 4. Clear Cell Renal Cell Carcinoma vs. Normal Gumz Renal, Clin Cancer Res, 2007 Lenburg Renal, BMC Cancer, 2003

2. Clear Cell Renal Cell Carcinoma vs. Normal 5 . Clear Cell Renal Cell Carcinoma vs. Normal Higgins Renal, Am J Pathol, $2003 \quad$ Yusenko Renal, BMC Cancer, 2009

3. Clear Cell Renal Cell Carcinoma vs. Normal Jones Renal, Clin Cancer Res, 2005

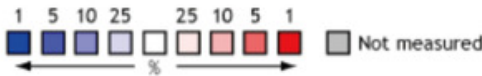

The rank for a gene is the median rank for that gene across each of the analyses.
The p-Value for a gene is its $p$-Value for the median-ranked analysis.
$\mathrm{E}$ OS NEDD4L + High + Low
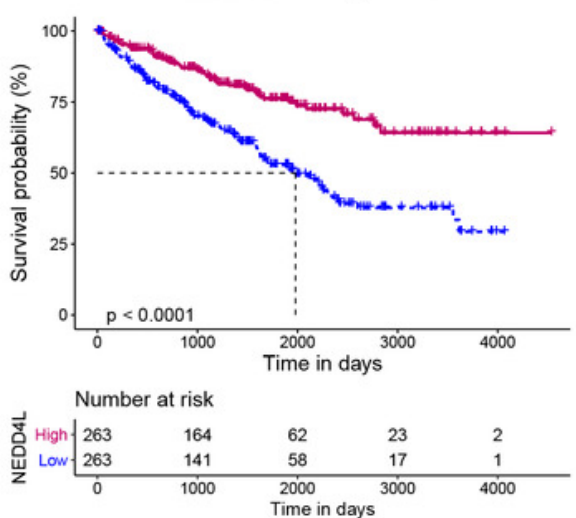

F

$F$
PFI
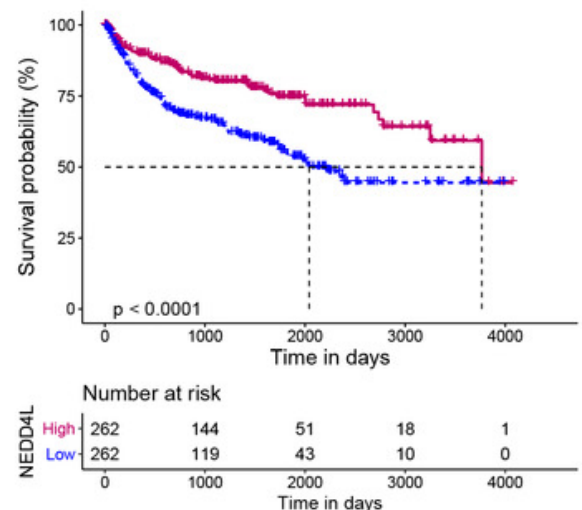

G

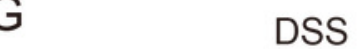

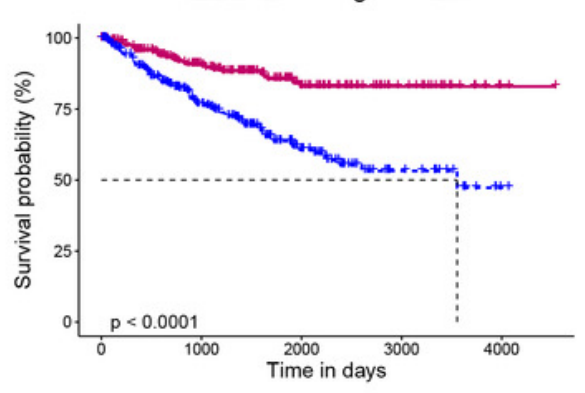

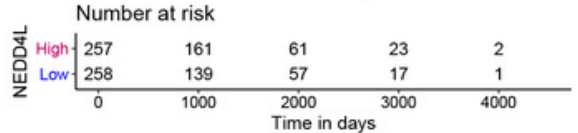


Figure 2

Figure 2. Prognostic analysis of NEDD4L in CCRCC patients grouped by sex, age, laterality, and cancer status

(A-D) Kaplan-Meier plot of the OS in high- and low-NEDD4L expression subgroups of CCRCC patients who were grouped according to sex (A), age (B), laterality (C), cancer status (D) 
A

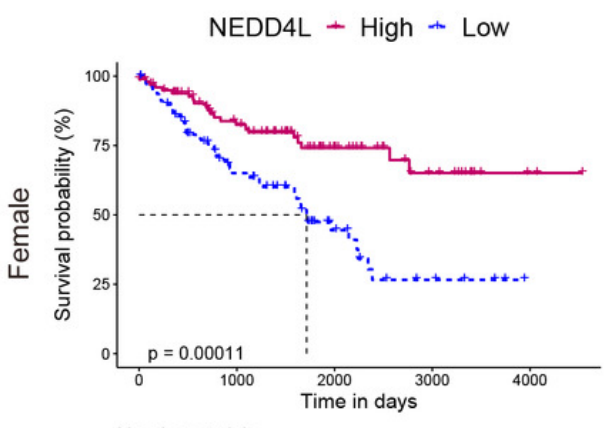

$\frac{\grave{\Phi}}{0}$

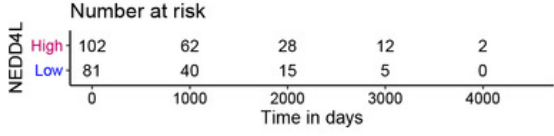

NEDD4L - High + Low
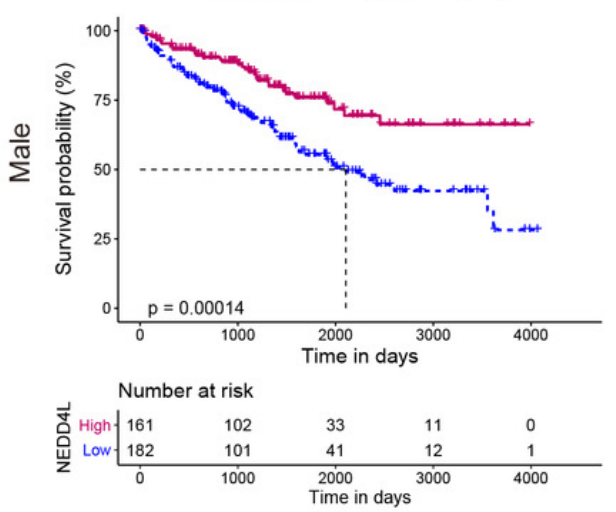

C

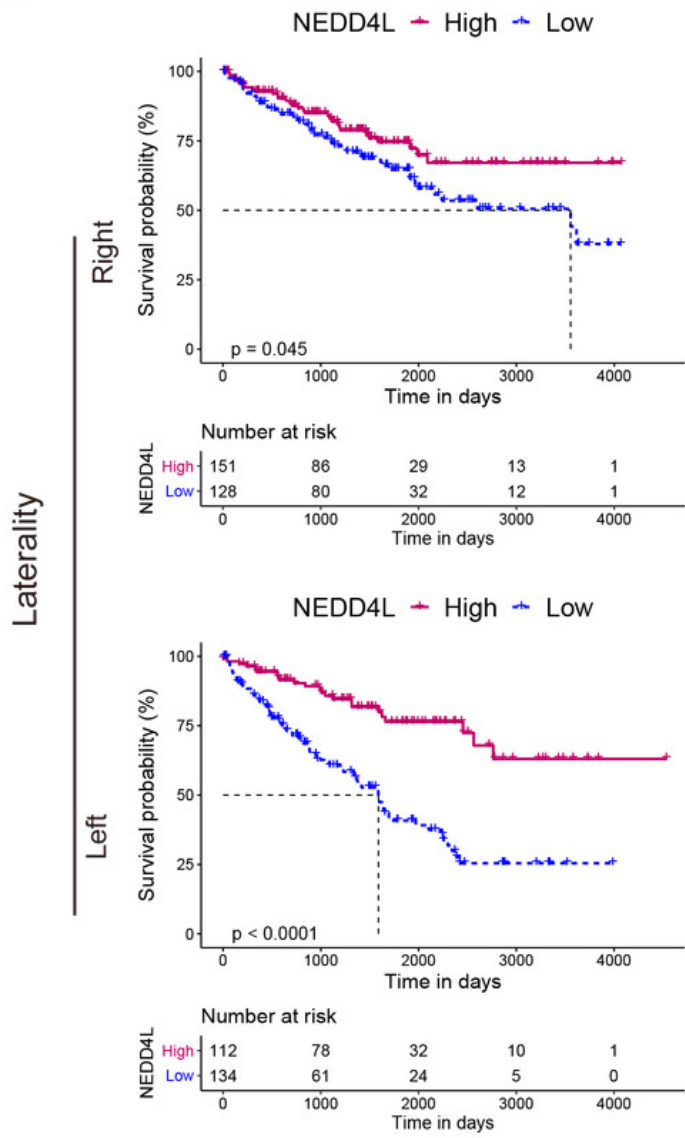

B

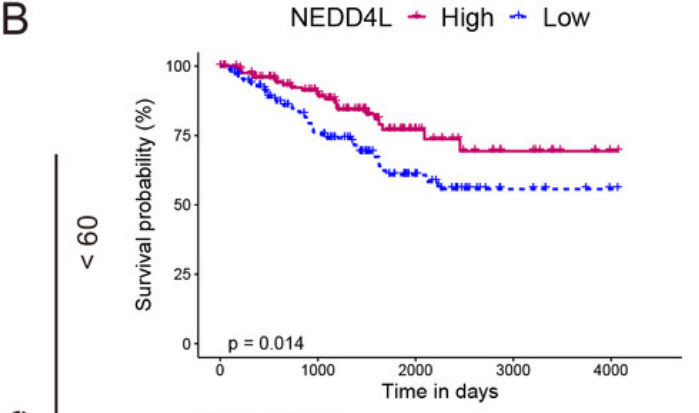

$\stackrel{8}{\mathbb{4}}$

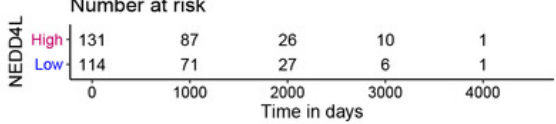

NEDD4L - High + Low
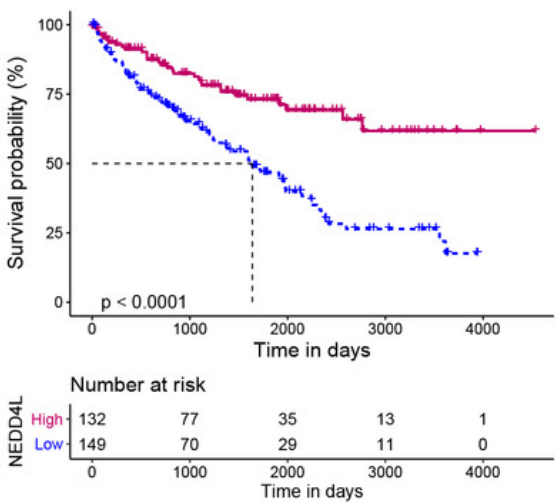

D

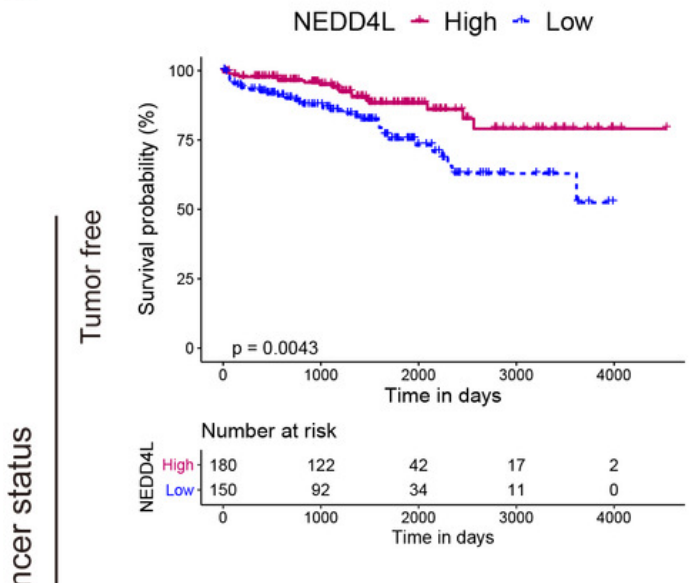

NEDD4L - High - Low
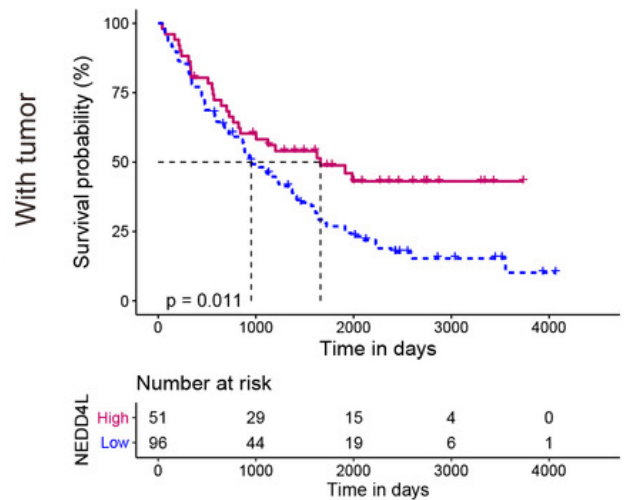


\section{Figure 3}

Figure 3. Prognostic analysis of NEDD4L in CCRCC patients grouped by histological grade, TNM stage, and tumor stage

(A-D) Kaplan-Meier plot of the OS in high- and low-NEDD4L expression subgroups of ccRCC patients who were grouped according to histological grade (A), TNM stage (B\&C), and tumor stage (D) 


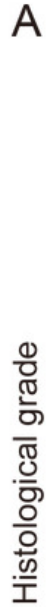

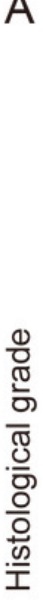

인
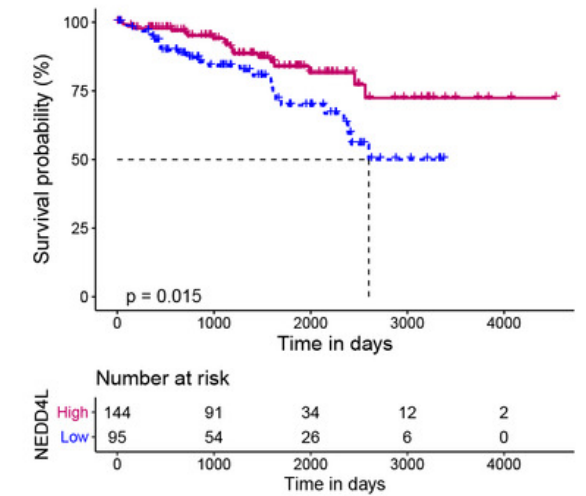

NEDD4L - High + Low
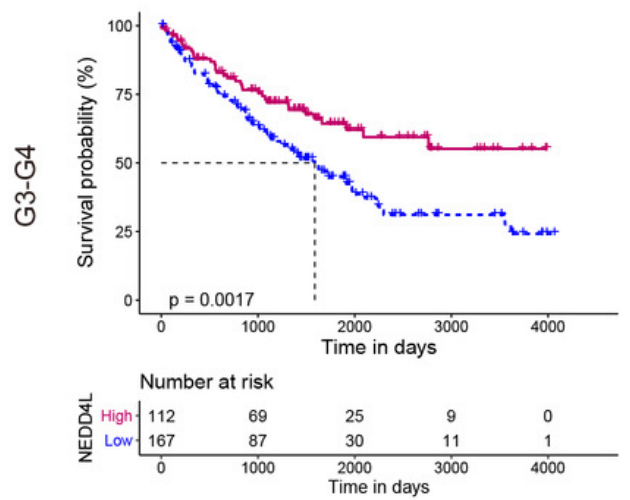

C

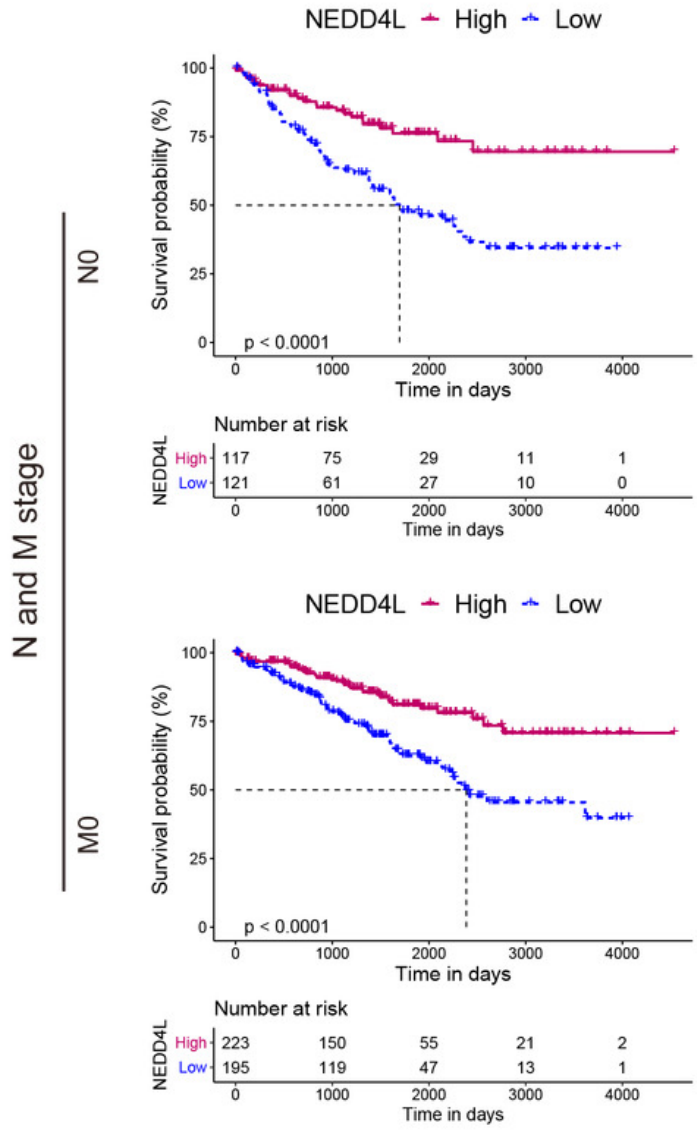

B

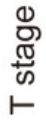
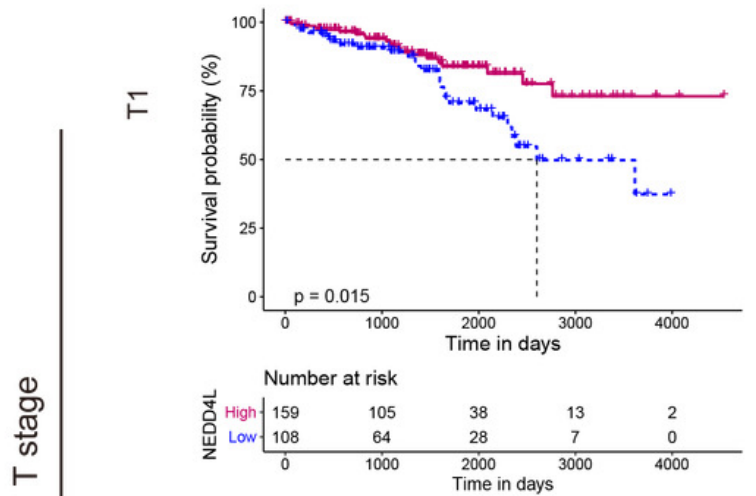

NEDD4L - High + Low
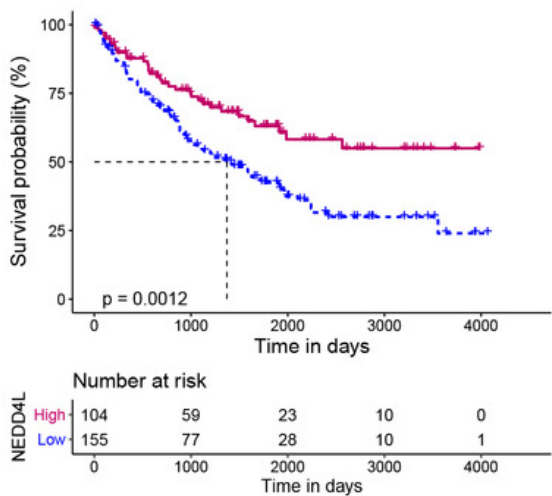

D
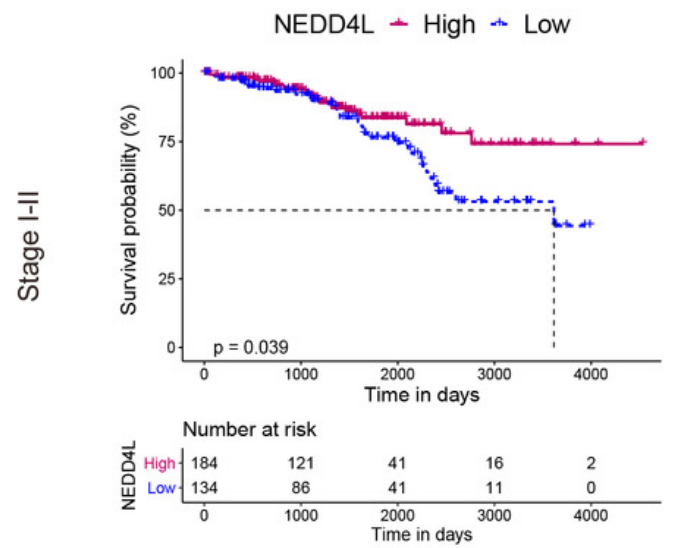

NEDD4L - High + Low
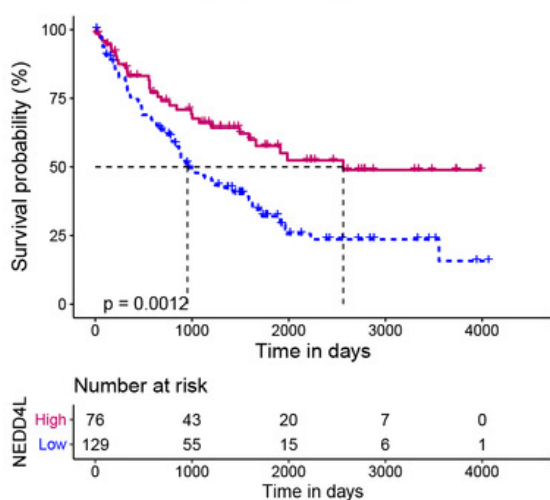
Figure 4

Figure $4 \mathrm{GO}$ and KEGG enrichment analysis of NEDD4L

(A) GO enrichment analysis of NEDD4L. (B) KEGG enrichment analysis of NEDD4L 
A

\section{GO analysis of NEDD4L-related genes}

\section{Quinone binding}

Palmitoyltransferase activity

NAD binding

Primary active transmembrane transporter activity ATPase-coupled transmembrane transporter activity Proton-transporting ATPase activity, rotational mechanism ATPase activity, coupled to transmembrane movement of ions, rotational mechanism ATPase-coupled ion transmembrane transporter activity ATPase-coupled cation transmembrane transporter activity Pyrophosphate hydrolysis-driven proton transmembrane transporter activity Bicellular tight junction Intrinsic component of mitochondrial membrane Integral component of mitochondrial membrane Apical junction complex

Proton-transporting V-type ATPase complex Proton-transporting two-sector ATPase complex Mitochondrial matrix Lipid oxidation Fatty acid oxidation Monocarboxylic acid catabolic process Fatty acid beta-oxidation Carboxylic acid catabolic process Organic acid catabolic process Fatty acid catabolic process Branched-chain amino acid metabolic process Branched-chain amino acid catabolic process Small molecule catabolic process

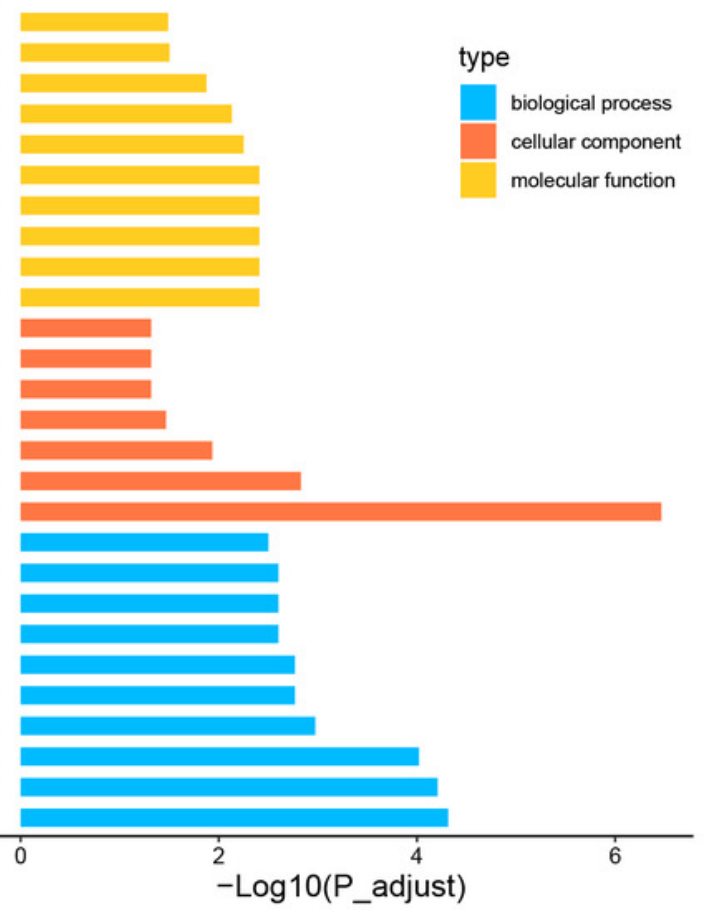

B

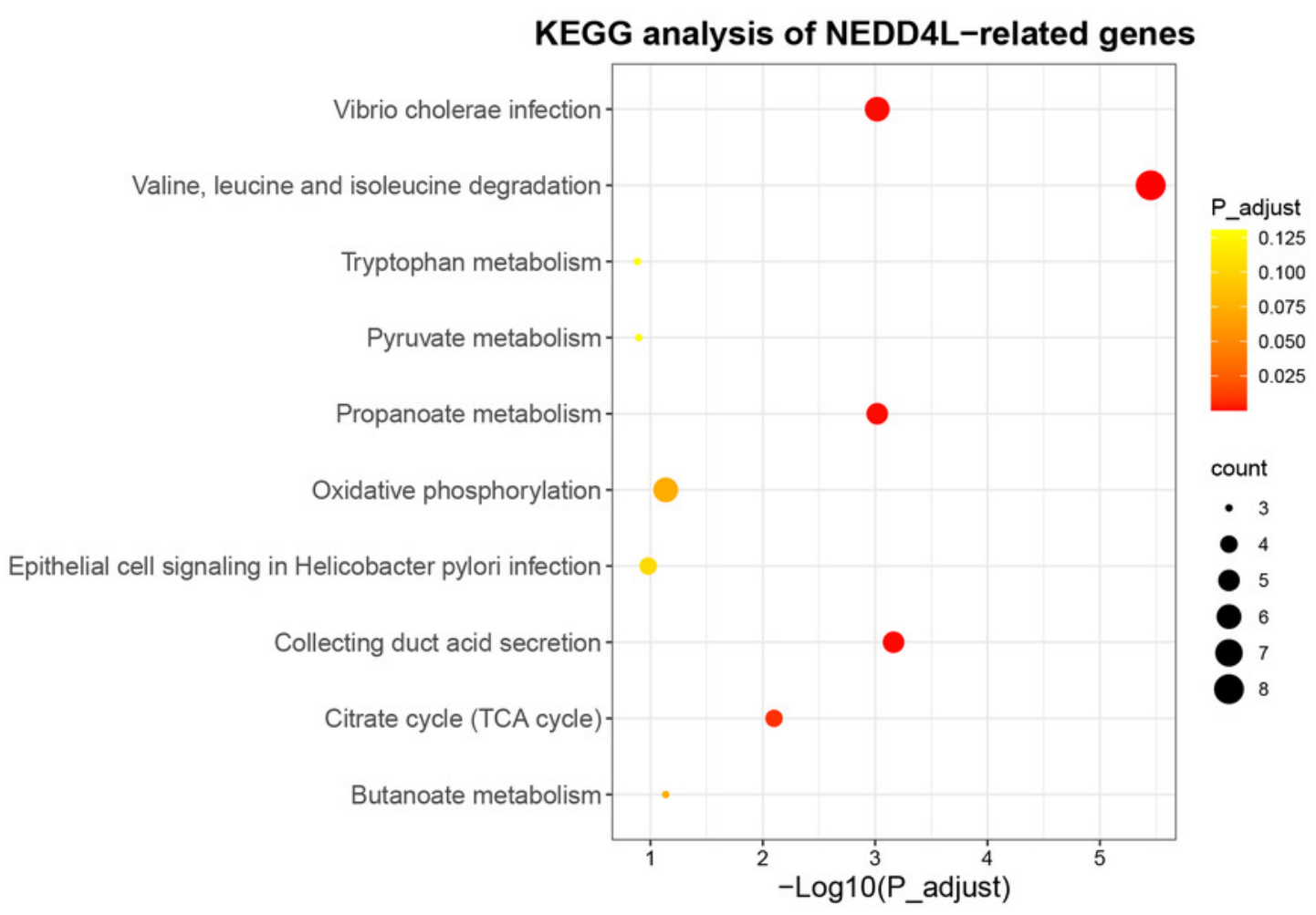


Figure 5

Figure 5. Construction of LASSO Cox regression model

(A-C) A LASSO Cox regression model was developed from NEDD4L and 183 related genes, calculating the tuning parameter $(\lambda)$ based on the partial likelihood deviance with tenfold cross-validation. Each dot represents a patient, and the heatmap represents the genes expression levels. The optimal $\log \lambda$ value is indicated by the vertical black line in the plot.

A

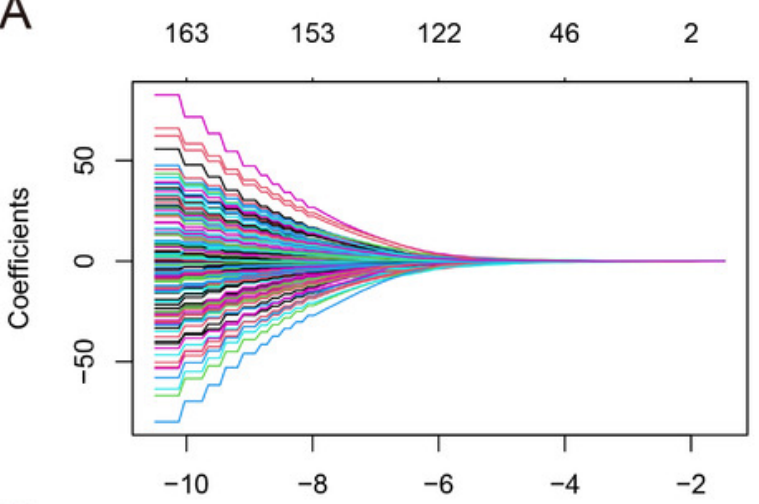

B

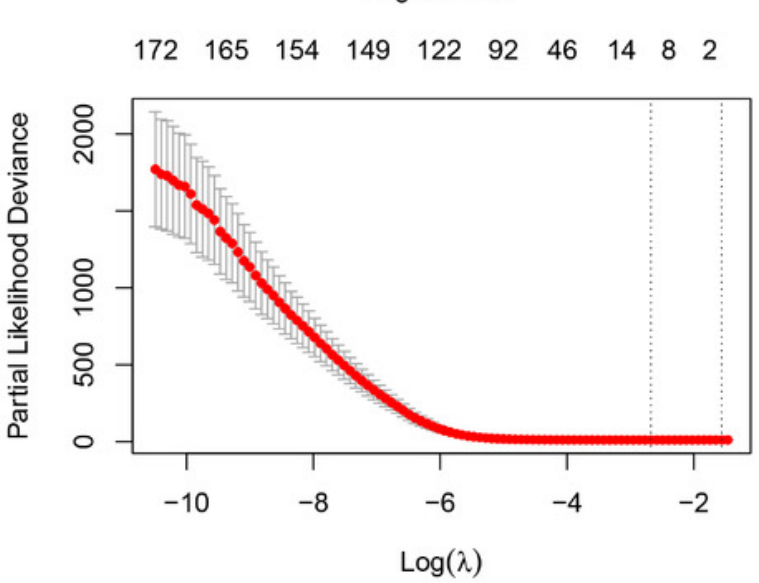

C
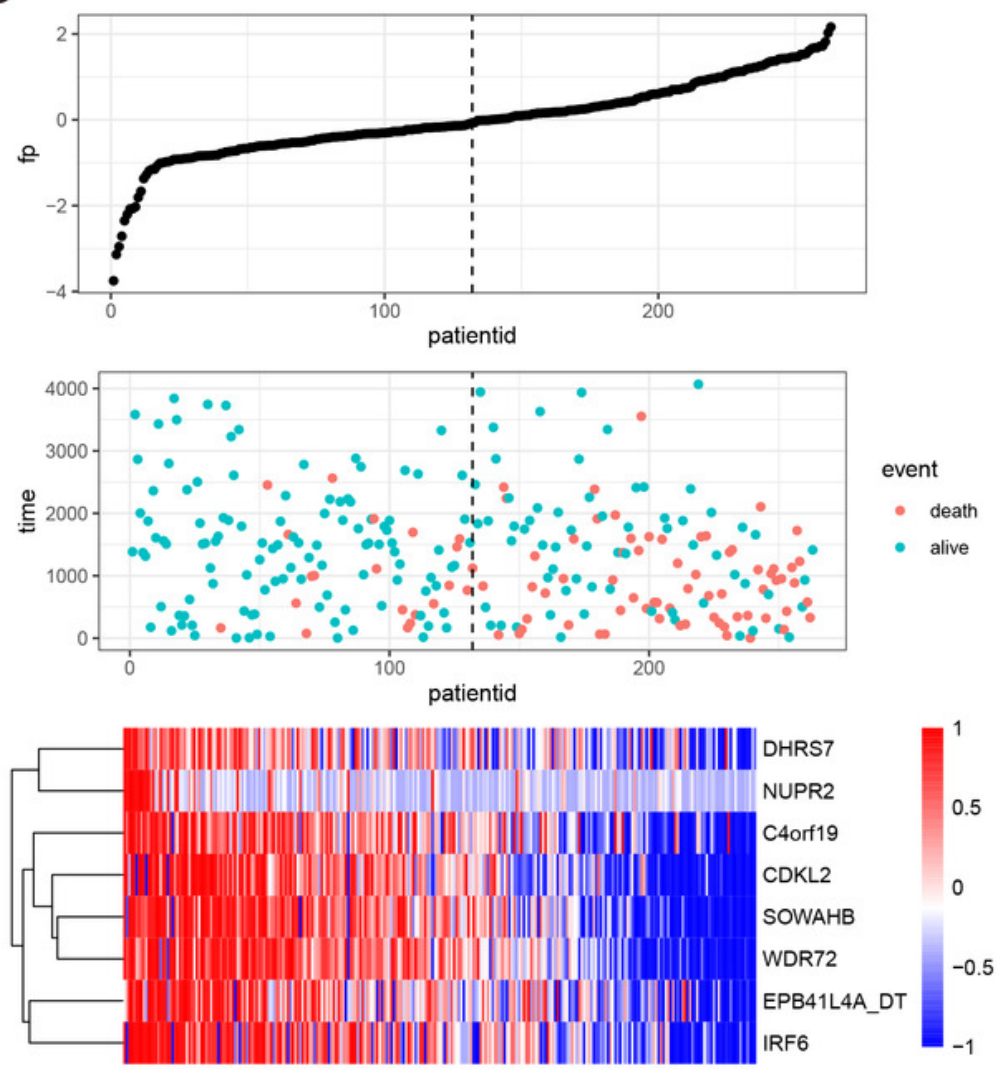
Figure 6

Figure 6. Reliability verification of NEDD4L-related prognostic model

(A-C) Kaplan-Meier plot of the DDS in the high- and low-risk groups of the TCGA_KIRC cohort .

(D-F) Time-dependent ROC analysis of the risk score for the DDS and survival status in the

TCGA_KIRC cohort

A

A TCGA training dataset Risk score + High_risk - Low_risk
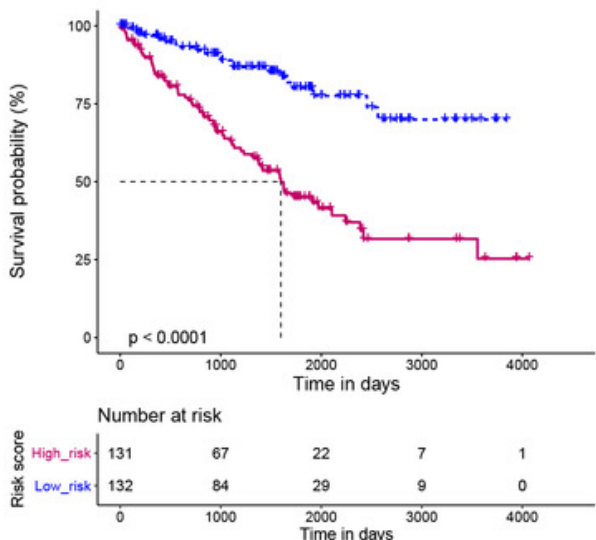

D TCGA training dataset

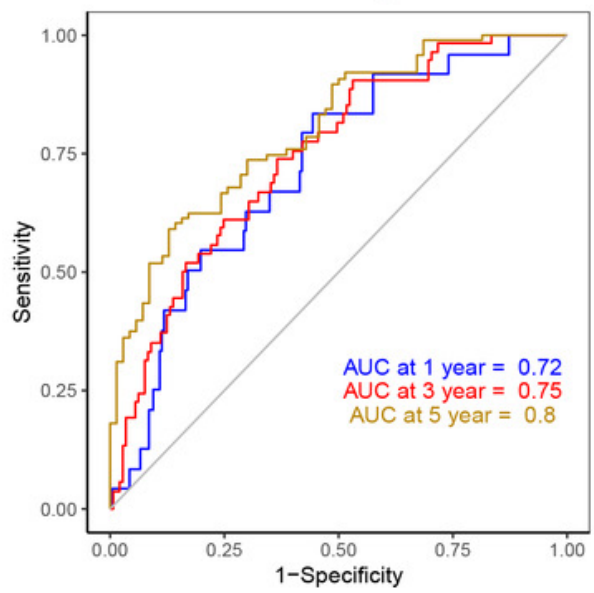

B

TCGA testing dataset

Risk score + High_risk + Low_risk
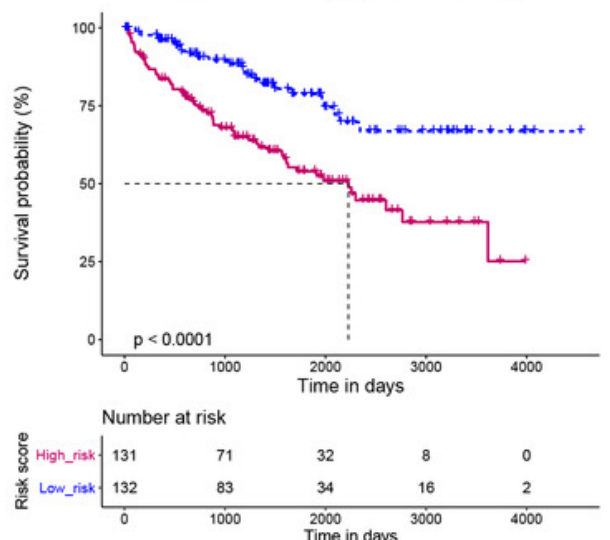

E TCGA testing dataset

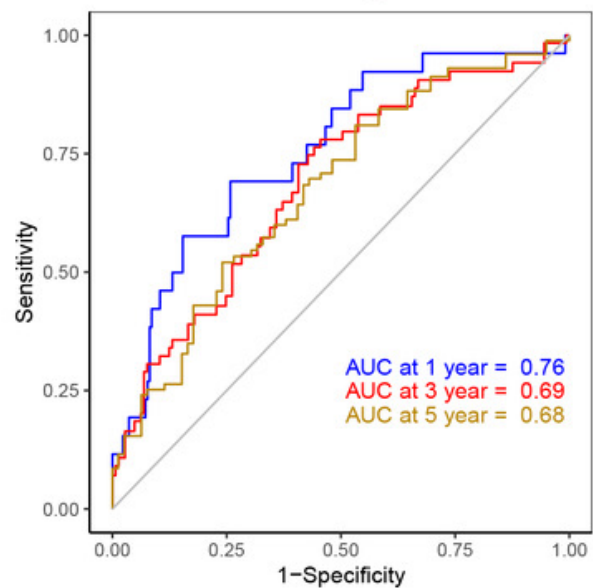

C

TCGA whole dataset

Risk score + High_risk + Low_risk
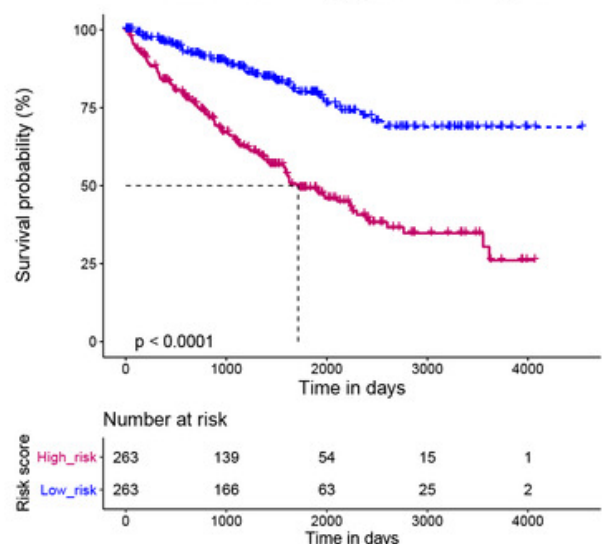

$\mathrm{F}$

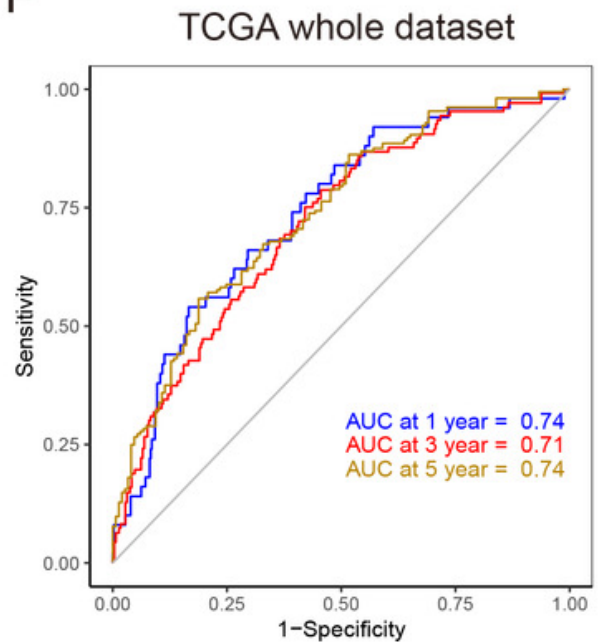


Figure 7

Figure 7. GSEA between $c C R C C$ patients with high and low NEDD4L expression in the TCGA_SKCM and GSE40435 cohorts (A-H). 
A

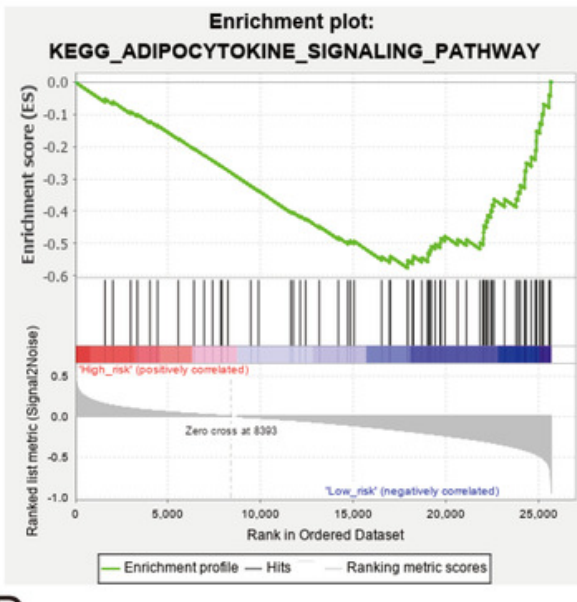

D

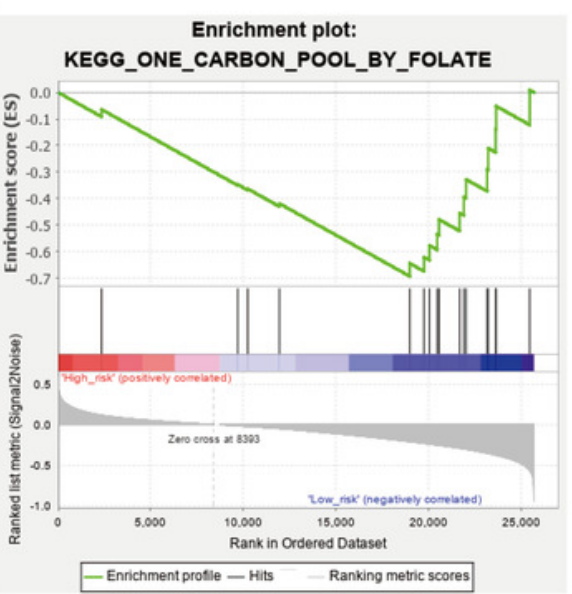

G

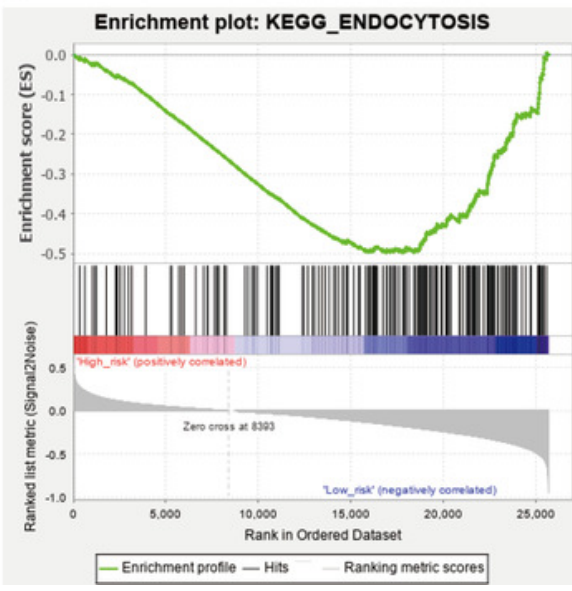

B

Enrichment plot: KEGG_RENAL_CELL_CARCINOMA

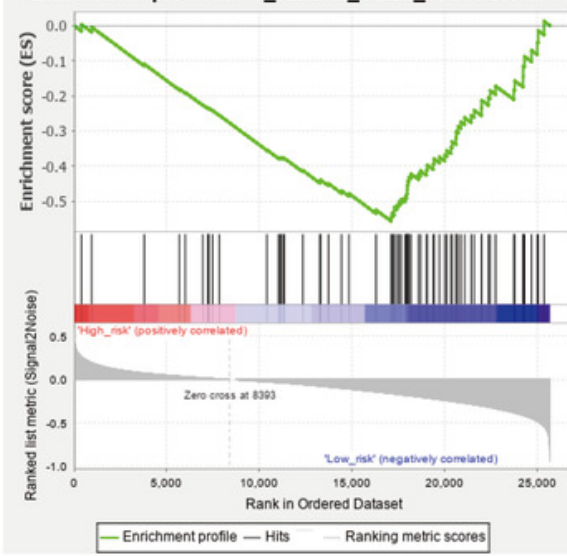

E

Enrichment plot: KEGG_INSULIN_SIGNALING_PATHWAY

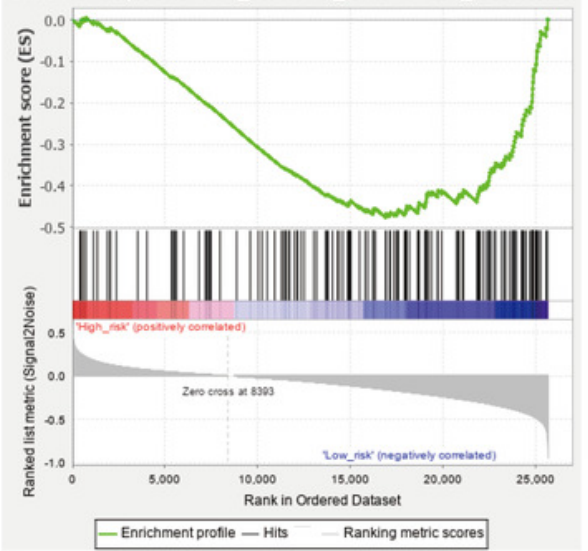

$\mathrm{H}$

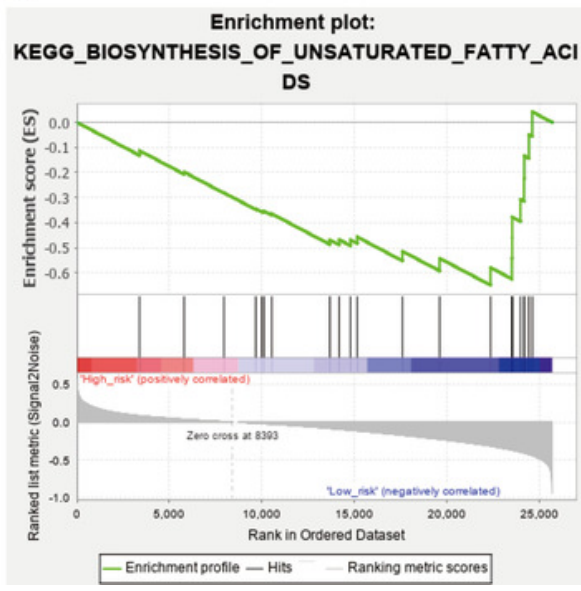

C

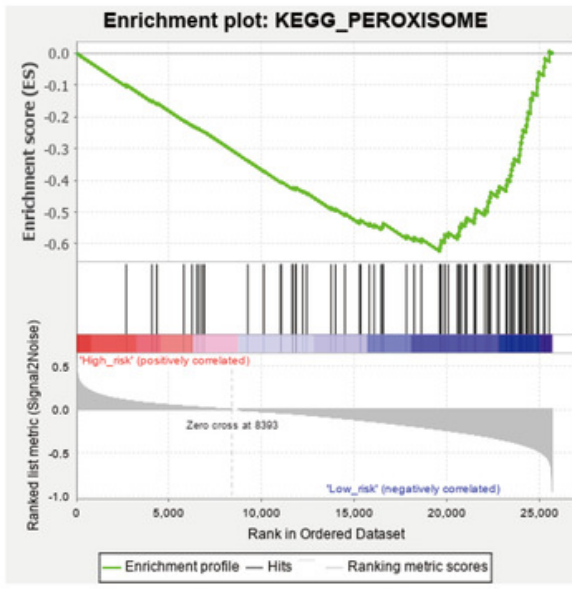

$\mathrm{F}$

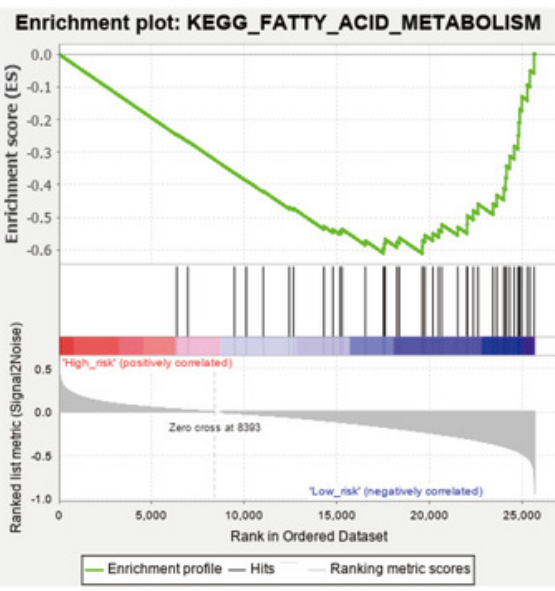




\section{Figure 8}

Figure 8. Integration of risk score and clinical characteristics

(A\&B) Univariate and multivariate regression analysis of the relation between the risk score and clinicopathological characteristics regarding OS in the TCGA_KIRC. (C) Nomogram developed to predict the 1-, 3-, and 5-year OS in the TCGA_KIRC data set 
A

Univariate Cox analysis in TCGA_KIRC

Factor No.sig Protective Risk

Risk_score (high risk vs. low risk

Laterality (right vs. left)

Histologic_grade (G3+G4 vs. G1+G2)

Stage (III-IV vs. I-II)

Gender (female vs. male)

Age (>=60 vs. $<60)$

B

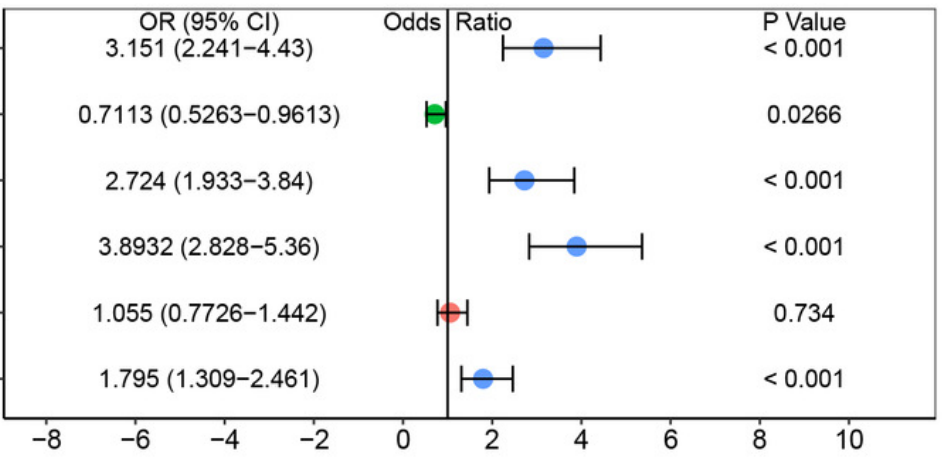

\section{Multivariate Cox analysis in TCGA_KIRC}

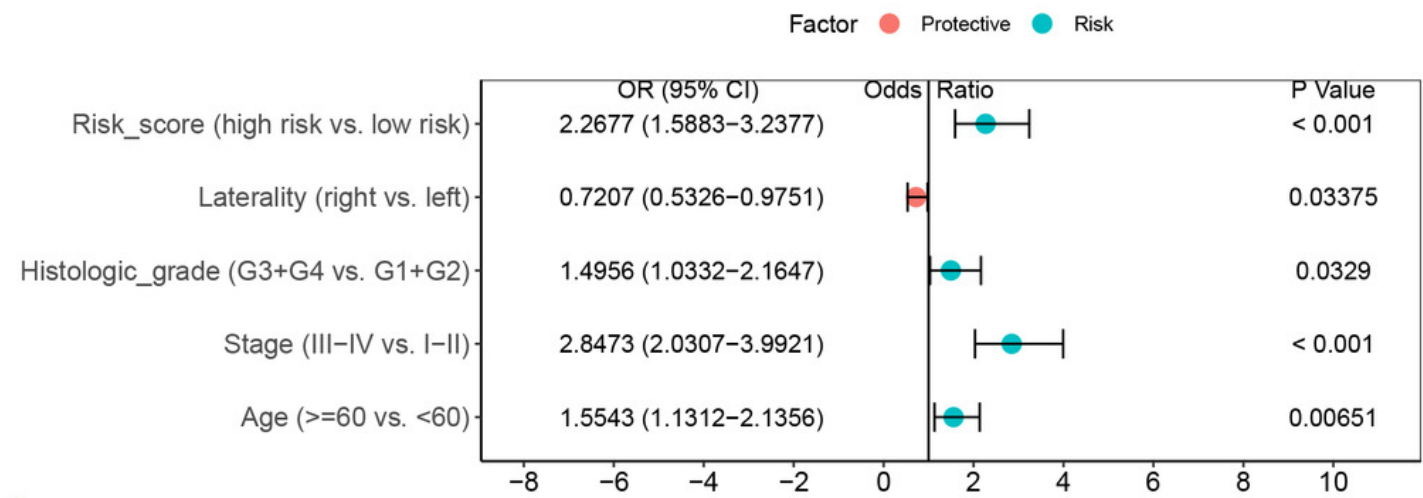

C

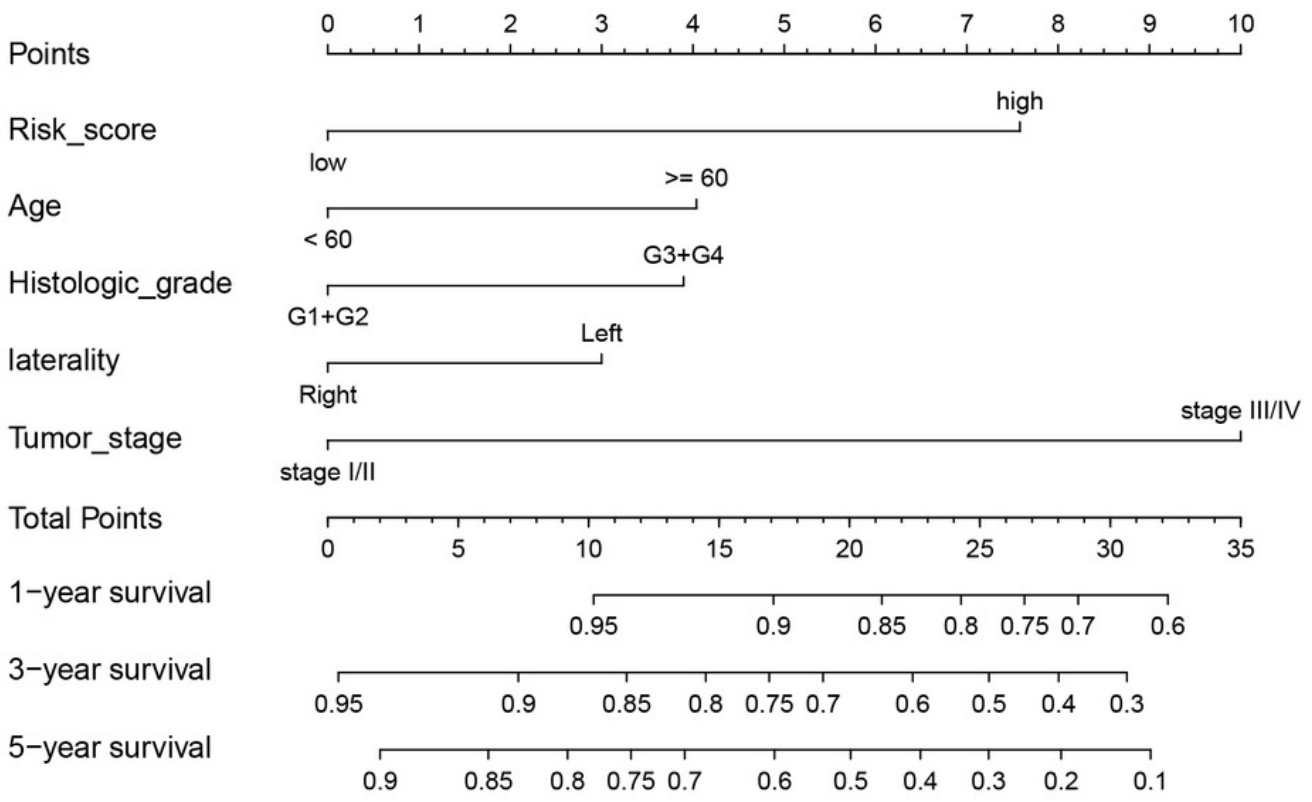




\section{Table 1 (on next page)}

Table 1 Univariate and multivariate analysis of overall survival in TCGA patients with cCRCC 
1 Table 1 Univariate and multivariate analysis of overall survival in TCGA patients with ccRCC

\begin{tabular}{|c|c|c|c|c|}
\hline \multirow{2}{*}{ Variables } & \multicolumn{2}{|c|}{ Univariate analyses } & \multicolumn{2}{|c|}{ Multivariate analyses } \\
\hline & Hazard ratio $(95 \% \mathrm{CI})$ & $p$-value & Hazard ratio $(95 \% \mathrm{CI})$ & $p$-value \\
\hline Age $(<60 v s . \geq 60)$ & $0.557(0.406-0.746)$ & $<0.001$ & $0.681(0.487-0.953)$ & 0.025 \\
\hline Sex (female $v s$. male) & $1.056(0.773-1.442)$ & 0.734 & & \\
\hline Laterality (left $v s$. right) & $1.409(1.042-1.904)$ & 0.026 & $1.345(0.972-1.860)$ & 0.073 \\
\hline NEDD4L (low $v s$. high) & $2.323(1.683-3.208)$ & $<0.001$ & $1.905(1.330-2.730)$ & $<0.001$ \\
\hline $\mathrm{T}(\mathrm{T} 1$ vs. T2/3/4) & $0.336(0.240-0.470)$ & $<0.001$ & $1.328(0.707-2.495)$ & 0.378 \\
\hline $\mathrm{N}(\mathrm{N} 0$ vs.N1/X) & $1.106(0.819-1.493)$ & 0.511 & & \\
\hline $\mathrm{M}(\mathrm{M} 0 v s . \mathrm{M} 1 / \mathrm{X})$ & $0.265(0.195-0.362)$ & $<0.001$ & $0.828(0.558-1.231)$ & 0.351 \\
\hline Cancer status (tumor free $v s$. with tumor) & $0.195(0.140-0.273)$ & $<0.001$ & $0.334(0.225-0.495)$ & $<0.001$ \\
\hline Stage (stage I vs. stage $\mathbb{I} / \mathbb{I I} / \mathrm{IV}$ ) & $0.253(0.183-0.349)$ & $<0.001$ & $0.447(0.240-0.834)$ & 0.011 \\
\hline Grade (G1/2 vs. G3/4) & $0.374(0.265-0.529)$ & $<0.001$ & $0.759(0.508-1.134)$ & 0.178 \\
\hline
\end{tabular}

2 
Table 2 (on next page)

Table 2 NEDD4L expression associated with clinical characteristics 
1 Table 2 NEDD4L expression associated with clinical characteristics

\begin{tabular}{lccc}
\hline Clinical characteristics & Total $(\mathrm{N})$ & OR $(95 \%$ CI $)$ & $P$ \\
\hline T (T1 vs. T2/3/4) & 526 & $0.456(0.322-0.646)$ & $<0.001$ \\
M (M0 vs. M1/X) & 524 & $0.530(0.342-0.821)$ & 0.004 \\
N (N0 vs.N1/X) & 526 & $1.063(0.754-1.499)$ & 0.726 \\
Stage (stage I / I vs. stage II /IV) & 523 & $0.429(0.299-0.615)$ & $<0.001$ \\
Grade (G1/2 vs. G3/4) & 523 & $0.456(0.321-0.647)$ & $<0.001$ \\
Sex (female vs. male) & 526 & $0.702(0.490-1.008)$ & 0.055 \\
Age (<60 vs. $\geq 60)$ & 526 & $0.771(0.547-1.087)$ & 0.137 \\
Laterality (left $v s$. right) & 525 & $1.411(1.000-1.991)$ & 0.049 \\
Cancer status (tumor free vs. with tumor) & 477 & $0.443(0.296-0.662)$ & $<0.001$ \\
\hline
\end{tabular}

2 OR: odds ratio, $\mathrm{CI}$ : confidence interval

$3 P$ values were calculated from two-sided chi-square test 


\section{Table 3 (on next page)}

Table 3 GSEA of the NEDD4L-related low risk group in TCGA_KIRC 
Table 3 GSEA of the NEDD4L-related low risk group in TCGA_KIRC

\begin{tabular}{cccc}
\hline MSigDB collection & Gene set name & NES & NOM p- \\
& & value \\
\hline & ERBB signaling pathway & 1.812 & 0.002 \\
& Vasopressin regulated water reabsorption & 1.713 & 0.012 \\
c2.cp.v6.2.symbols. & Propanoate metabolism & 1.708 & 0.041 \\
gmt & Neurotrophin signaling pathway & 1.660 & 0.008 \\
& Ubiquitin mediated proteolysis & 1.653 & 0.018 \\
& RNA degradation & 1.650 & 0.008 \\
\hline
\end{tabular}

2 NES: normalized enrichment score; NOM: nominal; FDR: false discovery rate. Gene sets with NOM p-value $<0.05$ and FDR q-value $<0.25$ are considered 3 as significant

4

5

6 